Aus der chirurgischen Poliklinik des Seraphimer-Iazaretts Stockholm.

\title{
Beiträge zur Behandlung der Hauttumoren, besonders dem Hautkrebs, mit Kohlensäureschnee.
}

Von Gunnar Nyström, Privatdozent.

(Mit 27 Abbildungen.)

1)ie Gefrierbehandlung mit Kohlensäureschnee hat bekanntlich während der letzten Jahre unter den Dermatologen immer größere Verbreitung gewonnen, da sie sich als eine bei gewissen Krankheiten sehr wertvolle Erweiterung des therapeutischen Rüstzeuges erwiesen hat.

Besonders gilt dies von dem Grenzgebiet der Chirurgie, und die Kohlensäurebehandlung ist daher auch für den Chirurgen von nicht geringem Interesse.

In der chirurgischen Poliklinik des Seraphimer-Lazaretts in Stockholm wurde mit der Anwendung der Wethode Ende I9Io begonnen, und etwa I50 Fälle, hauptsächlich Pigmentnaevi, Warzen, Angiome und Hautkrebs, sind mit derselben behandelt worden.

Im großen und ganzen ist also meine Erfahrung betreffs der Methode nicht sehr reich. Wo sie nur die auf weit größeres Material gegründeten Angaben anderer Forscher bestätigen, werde ich nur in größter Kürze die in unserer Klinik erhaltenen Resultate erwähnen, um der Hauptsache nach meine Darstellung cinigen Punkten zu widmen, in denen meine Studien möglicherweise zu einer Erweiterung unserer Kenntnis von der Anwendbarkeit der Methode beitragen können.

Was die Technik betrifft, so sind unsere Vorrrichtungen zur Herstellung von Kohlensäurestäbchen die denkbar einfachsten. Der Kohlensäurezylinder ist mit dem Hahn nach unten in einem einfachen Gestell aus Bandeisen placiert. Der Kohlensäureschnee wird in einem kleinen Beutel aus dichtem Samet aufgesammelt, 
der mittels einer im Rande des Beutels laufenden Schnur um das Mundstück des Zylinders geknüpft wird. In einer Metallröhre wird er dann in Stäbchen von gewünschtem Kaliber gepreßt. Der Hauptsache nach also dieselbe Technik, wie sie von $\mathrm{Sut}$ t on $\mathrm{u}$. a. beschrieben worden ist.

Die physikalische Wirkung der Kohlensäurebehandlung ist, soweit ich aus der Literatur habe ersehen können, bisher nicht Gegenstand näheren Studiums gewesen; da indessen die Kenntnis derselben von Bedeutung sowohl für die Indikation der Behandlung, wie für die Ausführung derselben ist, habe ich einige Untersuchungen betreffs der wichtigsten der diesbezüglichen Fragen, nämlich der Tiefenwirkung des Gefrierens, angestellt.

Der Kohlensäureschnee hat cine Temperatur von $-79^{\circ}$, und er ruft unmittelbar auf dem Körperteil, mit dem er in Berührung kommt, eine Temperaturerniedrigung hervor, deren Grad in einem gewissen Punkte des Körperteils von ciner ganzen Menge verschiedener Faktoren abhängt, nämlich dem Temperaturunterschied zwischen der Kohlensäure und dem betreffenden Körperteil (dessen Temperatur ja wechseln kann), der Zeit der Applikation, der Größe der Applikationsfläche und dem Abstande von derselben, der Dicke und dem Flüssigkeitsgehalt der verschiedenen an dem Aufbau des Körperteils beteiligten Gewebe, ihrer Wärmeleitfähigkeit in nichtgefrorenem und in gefrorenem Zustande, ihrer spezifischen Wärme und der Schmelzwärme der in den Geweben enthaltenen Flüssigkeit sowie endlich der Blutzirkulation. I) einige dieser Faktoren nicht konstant, sondern Schwankungen unterworfen sind, die nicht bestimmt werden können, so ist eine exakte theoretische Berechnung der Tiefenwirkung des Gefrierens nicht möglich, und auch eine nur einigermaßen annähernde Berechnung ist jedenfalls eine sehr komplizierte Aufgabe, auf die ich nicht eingehen kann.

Für rein praktische Zwecke gewähren indessen einige einfache empirische Versuche hinreichende Aufschlüsse. Aus ihnen geht hervor, daß ein kräftiger Druck bei der Applikation große Bedeutung für das Hinabdringen des Gefrierens in die Tiefe besitzt, was auch in früheren Publikationen betont worden ist. Wird ein Stück Kohlensäureschnee ganz lose auf die Haut 
gelegt, so dringt das Gefrieren im Laufe einiger Minuten kaum durch die Epidermis hindurch. Die heftige Gasbildung bewirkt eine Isolierung, der Druck ist also vor allem notwendig, um einen guten Kontakt mit der behandelten Partic zustande zu bringen. Ferner vermindert die Kompression durch A n ämisierung des darunterliegenden Gewebes die Wärmezufuhr durch die Zirkulation und fördert auch durch Auspressen von Lymphe und Verdünnung der nächstliegenden Gewebsschichten den Zutritt des Gefrierens zu tieferen Gewebsschichten.

Wieweit hinab in die Tiefe kann nun ein Gefrieren der Gewebe mittels Kohlensäure bei starker Kompression und innerhalb der begrenzten Gebiete, diegewöhnlich bei einer

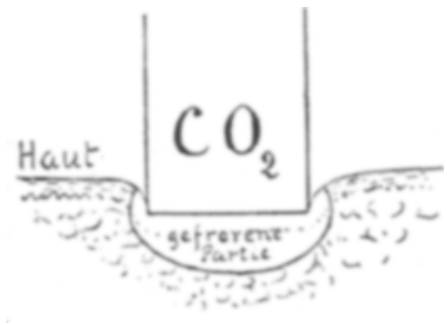

Fig. I Behandlung in Frage kommen, ausgeführt werden?

Ich habe diese Frage teils an lebendem Material - bei Versuchen, eine nahezu zentimeterdicke Hautmetastase von Krebs zu durchfrieren (siehe Fall Nr. I8, S. 574) - teils durch Experimente an Haut und Muskulatur von Leichen erörtert. Im ersteren Falle zeigte es sich unmittelbar nach einer 5 Minuten dauernden Applikation, daß das Gefricren ungefähr $6 \mathrm{~mm}$ in die Tiefe gedrungen war. Die letzteren Experimente wurden mit auf Körpertemperatur erwärmten Haut- und Muskelparticn ausgeführt, gegen die Kohlensäurestäbe verschiedenen Kalibers während I-IO Minuten gepreßt wurden. Sie ergaben folgende Resultate.

Zunächst zeigte es sich, daß das Gefrieren entsprechend dem $Z$ entrum des Stabes tiefer $\mathrm{h}$ in a bdringt, sehr natürlich übrigens, da an den Rändern die Ableitung nach den Seiten hin in entsprechendem Grade dic Tiefenwirkung vermindern muß. Im Durchschnitt zeigt die gefrorene Hautpartic die Kontur, wie sie aus Figur I ersichtlich ist.

Was nun dic gröBte Ticfe betrifft, bis zu welcher das Gefrieren hinabdringt, so hängt dies natürlich in erster Linie 
von der Applikationszeit ab, was aus nachstehender Tabelle näher hervorgeht (Zahlen nach halben Millimetern abrundet).

Das Ende des $\mathrm{CO}_{2}^{-}$ Stabes gegen die Haut gedrückt während

$\begin{array}{rr}1 & \text { Min. } \\ 2 & \\ 3 & \\ 4 & \\ 5 & \prime \\ 10 & \end{array}$

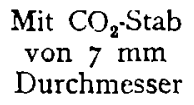

$\begin{array}{ll}2 & \mathrm{~mm} \\ 2 & \quad \\ 2,5 & \Rightarrow \\ 3 & " \\ 3,5 \quad " \\ 4 \quad "\end{array}$

Mit $\mathrm{CO}_{2}$-Stab von $17 \mathrm{~mm}$ Durchmesser

$\begin{array}{rl}4 & \mathrm{~mm} \\ 4,5 & \\ 5 & \Rightarrow \\ 5,5 & " \\ 6 & \Rightarrow \\ 6,5 & ;\end{array}$

Mit $\mathrm{CO}_{2}-\mathrm{Stab}$
von $34 \mathrm{~mm}$
Durchmesser

$5 \mathrm{~mm}$

$7, \tilde{j}$,

8,5 I0

Gefrieren von Muskulatur crgab hicrmit übereinstimmende Resultate.

A ls o: wenn z. B. das Gefrieren von einem Stabe von I7 mm Durchmesser aus während der ersten Minute in eine Tiefe von $4 \mathrm{~mm}$ dringt, crreicht es nach 5 Minuten $6 \mathrm{~mm}$, überschreitet aber diese Tiefe nach weiteren 5 Minuten nur mit 0,5 mm. Das Eindringen in die Tiefe nimmt also nach den ersten Minuten schnell ab, und praktisch wird das Maximum schon nach $5 \mathrm{Mi}$ nuten erreicht.

Aus der Tabelle geht indessen noch eine andere Sache hervor, die nämlich, daß die Größe der auf einmal behandelten Fläche eine wichtige Rolle für den Effekt des Getrierens spielt, indem die Tiefenwirkung desselben mit der Größe der Fläche wächst. So ist z. B. bei einer Applikationszcit von 3 Minuten die Gefrierticfe bei Applikationsflächen von 7 , I7 und $34 \mathrm{~mm}$ Durchmesser bzw. 2,5, 5 und $7 \mathrm{~mm}$. Hieraus ergibt sich die praktische Konsequenz, daß bei einer Behandlung, die auf größtmögliche Tiefenwirkung abzielt, eine größtmögliche Fläche auf einmal zu behandeln ist. Soweit ich habe finden können, hat man diesem Umstande nicht zuvor Beachtung geschenkt.

Verfolgen wir in der Tabelle den Wert der Gefriertiefe für zunehmende Applikationsflächen bei einer bestimmten Applikationszeit, so sehen wir, daß dieser Wert in langsamerer Progression zunimmt, als es der Zunahme des Durchmessers der Fläche entspricht. Von einer kreisförmigen Applikationsfläche von $7 \mathrm{~mm}$ Durchmesser aus erstreckt sich $z$. B. nach 5 Minuten 
das gefrorene Gebiet bis zu einer Tiefe entsprechend etwa der Hälfte des Durchmessers der Fläche, während für eine Applikationsfläche von I7 mm das entsprechende Verhältnis etwa I : 3 und für cine Fläche von $34 \mathrm{~mm}$ etwa $I: 4$ ist.

Auch durch bedeutende Steigerung der Größe der Gefrieroberfläche kann demnach das Gefrieren nicht beträchtlich weiter nach der Tiefe zu getrieben werden, als wie es die oben angeführten Versuche zeigen.

Innerhalb der gefrorenen Partie ist die Temperatur natürlich nicht überall die gleiche, sondern sie steigt kontinuicrlich von der Temperatur $-79^{\circ}$ der Kohlensäure an der Applikationsfläche bis zum Gefrierpunkt der Gewebssäfte, der etwas unter $0^{0}$ (Gefrierpunkt des Blutes -o,56) liegt, an der unteren Grenze der gefrorenen Partic.

Nach diesen physikalischen Vorerörterungen wenden wir uns den physiologischen Wirkungen des Kohlensäuregefricrens zu.

Der Grad der Wirkung hängt ab I. von der Intensität des Gefrierens, d. h. dem Grade der Temperaturerniedrigung, 2. von der Zeit, während welcher diese Temperaturerniedrigung gewirkt hat, 3. von der Empfindlichkeit des Gewebes für Kälte.

Aus dem oben Angeführten geht hervor, daß die Inten $s$ it ät des Gefrierens sehr rasch nach der Tiefe zu abnimmt, so da $B$ dessen Wirkung (bei den Voraussetzungen, dic bei seiner therapeutischen Anwendung statthaben) verhältnismäBig o b e r flä chli ch bleibt. Für eine bestimmte Gewebsschicht, die nicht direkt an der Oberfläche selbst liegt, z. B. das Rete Malpighii, hängt die Intensität des Gefrierens in hohem Grade von der Tiefe $a b$, bis $\mathrm{zu}$ welcher das Gefrieren getrieben wird, wobei die Temperaturerniedrigung natürlich um so stärker wird, je oberflächlicher im Verhältnis zur Gesamttiefe des Gefrierens die fragliche Gewebsschicht liegt.

Die Gefriertiefe wiederum hängt, wie oben gezeigt worden ist, von dem Drucke und der GröBeder Gefrierflä che sowie von der Applikationszeit ab. Es ist hier auch auf die Rolle hinzuweisen, weiche die $B$ esch a f $f$ en heit der $\mathrm{H}$ a u t spielen muB; eine je dickere Hornschicht die Epi- 
dermis hat, um so gröBer ist der Abstand von der Applikationsfläche bis hinab zu den lebenden Gewebsschichten, und um so geringer ist die Einwirkung auf diese. Die Hornschicht scheint auch dem Gefrieren bedeutend gröBeren Widerstand $\mathrm{zu}$ bieten als die übrigen Teile der Haut. So kann es z. B. geschehen, daß man bei 2 Minuten dauernder Behandlung einer stark hornigen Warze kein anderes Resultat als eine unbedeutende Hyperämie am Rande erhält, während die angrenzende Haut nach I 5 Sekunden Gefrieren bereits Blasenbildung ergibt. Bei einem und demselben Individuum erhält man z. B. kräftige Blasenbildung nach 20 Sekunden Applikation auf der Volarseite des Unterarms, dagegen kaum eine Veränderung nach ebenso lange dauernder Applikation. auf den Volarseiten der Fingerspitzen.

Neben der Intensität des Gefrierens ist die A p p $1 \mathrm{i}$ k a t i o n s$z$ e it von entscheidender Bedeutung für die physiologische Wirkung. Eine Eisblase, die während einiger Minuten direkt auf die Haut gelegt wird, verursacht nur eine leichte Hyperämie, kann aber bekanntlich bei länger dauernder Einwirkung eine oberflächliche Nekrose hervorrufen. Auf normaler, weicher Haut einer erwachsenen Person, z. B. auf dem Arm, bewirkt eine Applikation von fester Kohlensäure unter kräftigem Druck während 5 Sekunden Hyperämie, begleitet von einer nach einigen Stunden verschwindenden urticariaähnlichen Anschwellung, während I5 bis 30 Sekunden eine oberflächliche Blasenbildung in der Epidermis, während I Minute oder mehr vollständige Nekrose der Epidermis.

Endlich ist auch die Empfindlichkeit des behandelten Gewebes $\mathrm{f} \ddot{\mathrm{u}} \mathrm{r} \mathrm{K}$ ält e von nicht geringer Bedeutung. Das A 1 t e $r$ übt hier einen wesentlichen Einfluß aus. Die Haut bei Erwachsenen ist bedeutend widerstandskräftiger als die Haut bei Kindern. Bei einem einige Monate alten Kinde erhielt ich nach ro Sekunden dauernder Behandlung eines Angioms am Unterarm eine vollständige Nekrose der Haut, so daß ich es für das zweckmäßigste erachtete, das Stück zu excidieren und den Defekt zusammenzunähen. Eine Behandlung der entsprechenden Hautpartie während ebenso langer Zeit bei einem erwachsenen Menschen hat, wie oben erwähnt, nichts anderes als Hyperämie oder höchstens oberflächliche Blasenbildung zur 
Folge. Ich glaube auch Gründe zu der Annahme zu haben, daß innerhalb derselben Altersgruppen und bei dem Äußeren nach gleichartiger Hautbeschaffenheit verschiedene in d ivid u elle Empfindlichkeit eine Rolle spielt.

Fassen wir vom praktischen Gesichtspunkt aus die verschiedenen Umstände zusammen, die für die Wirkung des Kohlensäuregefrierens von Bedeutung sind, und mit denen man demnach bei der exakten Dosierung $z u$ rechnen hat, so sind es die folgenden:

I. der angewandte Druck,

2. die Größe der durchfrorenen Oberfläche,

3. die Beschaffenheit der Haut, besonders die Dicke der Hornschicht,

4. die Zeitdauer der Applikation und

5. das Alter (nebst individueller Disposition) des Patienten.

Einige Autoren, Juliusberg, $\mathrm{H}$ och haus, Nobl und $\mathrm{Springels}$, Salomon, haben die mikroskopischen Veränderungen untersucht, die von dem Kohlensäuregefrieren hervorgerufen werden; da aber ihre Angaben nicht völlig miteinander übereinstimmen, habe ich gleichfalls einige derartige Untersuchungen angestellt, teils an normaler Haut vom Menschen, teils an Haut von Kaninchen. Beim Studium der Präparate hat Herr Dr. G. $\mathrm{H}$ ed r é n, Professor der pathologischen Anatomie am Karolinischen Institut, freundliche Hilfe geleistet. Die Resultate teile ich in ihren Hauptzügen mit.

I. Haut von der Glutealgegend eines 6rjährigen Mannes, erhalten bei Excision einer bösartigen Geschwulst. 24 Stunden vor der Operation wurden mit Erlaubnis des Patienten 3 Flecke (von etwa I5 mm Durchmesser) der neben der Geschwulst befindlichen Haut mit Kohlensäure während bzw. I5, 30 und 60 Sekunden behandelt. Fixierung in Formalin und Alkohıl; Hämatoxylin-Eosinfärbung.

Die Präparate zeigen schlechte Färbung der Epidermis und eine Absprengung derselben vom Corium, um so ausgeprägter, je länger das Gefrieren gedauert hat, nach 60 Sekunden Gefrieren fast vollständig. In den kürzere Zeit gefrorenen Hautpartien finden sich hier und da ziemlich wohlgefärbte, noch am Corium festsitzende Basal zellen auch die abgesprengten Epidermispartien zeigen Flecke mit blaugefärbten Epithelzellen, die jedoch Degenerationserscheinungen aufweisen, wie Kernschrumptung und Vakuolenbildung. In der Epirler- 
mis werden polynukleäre Leukozyten angetroffen, um so zahlreicher, je länger das Gefrieren geclauert hat.

Das Exsudat unter der Basalschicht teilweise fibrinös, enthält zahlreiche polynukleäre Leukozyten; solche infiltrieren auch in große. Menge sowohl die Cutis als das subkutane Fett. Die Gefäßlumina im allgemeinen leer, ohne Anzeichen von Thrombose. In den 30 und 6n Sekunden lang gefrorenen Präparaten trifft man jedoch vereinzelte kleinere thrombotisierte Gefäße im Corium an.

2. Normale Bauchhautvon 20 jähriger Frau, excidiert im Zusammenhang mit einer Operation wegen chronischer Appendicitis. Mit Erlaubnis der Patientin wurde Gefrieren kleiner Flecke in der Ausdeknung des kinftigen. Schnittes ausgefïhrt.

A) 3 Flecke von etwa $8 \mathrm{~mm}$ Durchmesser, 20 Stunden vor der Excision bzw. I5, 30 and 60 Sekunden lang Gefrieren unterworfen. Die Hautstücke wurden in Formalin und Alkohol fixiert; HämatoxylinEosinfärbung.

Die Epidermis zeigt Veränderungen von derselben Art wie beim vorhergehenden Material, aber weniger ausgeprägt, indem die Zellen besser ihre Färbbarkeit beibehalten haben und die Absprengung nur auf einer Menge kleinerer Flecke eingetreten ist, gewöhnlich entsprechend den Vertiefungen zwischen den Papillen; außerdem wurden nur hier und da einige Leukozyten zwischen den Epidermiszellen angetroffen. Corium und subkutanes Fett sind mäßig mit polynukleären Leukozyten infiltriert. Nekrotische Teile von Schweißdrüsen werden hinab bis $z u \quad 2 \mathrm{~mm}$ Tiefe unter der Hautoberfläche angetroffen. In einem 30 Sekunden lang gefrorenen Präparat wurde auf der Grenze zwischen dem Corium und dem Fett eine thrombotisierte Arterie angetroffen, in deren Wandung die Zellkerne nicht Farbe angenommen hatten (Nekrose ?); im übrigen keine Thrombosen. In den oberflächlichsten Schichten des subkutanen Fettes oder unmittelbar unter dem Corium findet sich hier und da ein fcines, fibrinähnliches Netzwerk.

B) 3 Flecke von etwa $8 \mathrm{~mm}$ Durchmesser, 5 Tage vor der $\mathrm{Ex}$ cision bzw. I5, 30 und 60 Sekunden lang Gefrieren unterworfen. Fixierung der Hautstiicke in Formalin und Alkohol, Färbung mit Hämatoxy'in-Eosin.

Die Epidermis zeigt verschiedene Veränderungen. In dem $5_{5} \mathrm{Se}$ kunden lang gefrorenen Stück ist die Fpiciermis mit einer diünnen Kruste belegt, von normaler Dicke, schlechter gefärbt als normalerweise, mit aufgelockerter Basalschicht, die aber nirgends vollständig nekrotisch ist. In dem 30 Sekunden lang gefrorenen Stück ist der gröBte Teil der Epidermis nekrotisiert oder in Abstoßung begriffen; in der Mitte der gefrorenen Partie findet sich jedloch noch eine Haufe wohl gefärbter, am Corium festsitzender Basalzellen; Epidermisheilung von den Rändern aus unter der noch vorhandenen nekrotischen Epidermis. decke. In dem 60 Sekunden lang gefrorenen Stück findet sich kein 
Epidermisdefekt (schon fertige Heilung oder niemals vollständige Nekrose ?); die Epidermis ist unter den Rändern der noch vorhandenen dicken Kruste verdickt, besonders das Stratum germinativum, und stärker gefärbt als normal, unter dem Zentrum der Kruste dagegen stark verdünnt. Das Corium hat in sämtlichen Präparaten im allgemeinen normalen Zellengehalt, und die Leukozyteninfiltration ist verschwunden, hier und da gehen aber $Z$ üge von Granulationsgewebe mit neugebildeten Gefäßen, die aufwärts nach den Papillen zu oder in sie hinein verlaufen. In einem der Präparate werden nekrotische Schweißdrüsenpartien angetroffen. In allen Präparaten finden sich thrombotisierte Gefäße, sowohl im Corium als in der angrenzenden Schicht des subkutanen Fettes, in dem I5 Sekunden lang gefrorenen Stück zeigt jedoch die überwiegende Anzahl Gefäße vollständig freies Lumen. Unmittelbar unter dem Corium oder in den oberflächlichsten Schichten des subkutanen Fettes findet sich in allen Präparaten das auch unter A) beschriebene feine fibrinähnliche Netzwerk fleckweise oder in mehr zusammenhängender Schicht.

Haut von I und 2 Monate alten $\mathrm{K}$ aninchen, excidiert 24 Stunden nach Gefrieren mittels fester Kohlensäure während 30 und 60 Sekunden, zeigt keine Veränderungen von besonderem Interesse. Mehr oder weniger reichliche Blutungen sowie eine stärker hervortretende fibrinöse Exsudation ergeben von dem vorhergehenden Material abweichende Bilder. Bei einem 2 Monate alten Kaninchen scheint Gefrieren während 30 oder 60 Sekunden keine größere Zerstörung der Epidermis bewirkt zu haben, während bei einem I Monat alten Kaninchen fast die ganze gefrorene Epidermis von einer dicken Schicht Eiterkörper durchsetzt oder durch eine solche ersetzt worden ist.

Die Veränderungen, die ein Gefrieren mittelst Kohlensäure in fester Form während $5_{5}-60$ Sekunden in normaler Haut hervorruft, sind mithin der Hauptsache nach eine je nach der Dauer der Gefrierzeit mehr oder weniger hervortretende Auflockerung oder Zerstörung der Epidermis sowie Absprengung derselben von dem Corium, ent$z$ ündliche $Z$ elleninfiltration im Corium und subkutanen Gewebe, die binnen einiger Tage verschwindet, und anderen Stelle im Corium $Z$ ügevon Granulationsgewebe und neugebildeten GefäBentreten, Nekrosevon SchweiBdrüsenteilen, die hinab bis $z u 2 m m$ Tiefe unter der Hautoberfläche angetroffen werden, endlich Thromboseder Gefäße.

Juli usberg untersuchte Haut, die I4 Stunden vor der 
Excision mittels Kohlensäurespray 30 Sekunden lang gefroren worden war, und fand dabei Nekrose der Epidermis, reichliche Leukozyteninfiltration im Corium, Koagulation des Inhaltes der Lymphgefäße und Thrombose der Blutgefäße. Diejenigen von meinen Präparaten, die mit $\mathrm{Julius}$ berg zunächst zu verglcichen sind ( $\mathrm{I}$ und $2 \mathrm{~A}$, erhalten $24 \mathrm{bzw}$. 20 Stunden nach dem Gefrieren), zeigen im großen und ganzen übereinstimmende Bilder, weichen aber auffallend darin $a b$, daß die $\mathrm{Th}$ rombose äuBerst spärlich vorkommt im Gegensatz zu der reichlichen Thrombenbildung, die $\mathrm{Julius}$ berg beobachtet zu haben scheint. Erst nach einigen Tagen treten die Thrombosen nach meinen Präparaten in reichlicherer Menge auf.

In einer in diesem Jahre herausgegebenen Arbeit über Kohlensäurebehandlung von Angiomen und Naevi teilt S a lo mon auch die Resultate von mikroskopischen Untersuchungen excidierter Angiokavernome mit, die mit Kohlensäure ein- bis zweimal Io- 55 Sekunden lang behandelt worden waren. In den kutanen Angiomschichten zeigen die Gefäße hyaline Wandthromben, Degeneration, „Entzündung“ und Abstoßung des Endothels sowie eine lebhafte Regeneration von Endothelzellen, ausgesprochen vorhanden bereits 4 Tage nach dem Gefrieren, wodurch das Gefäß obliteriert wird. Derartige Veränderungen werden nur in der oberflächlichen Cutisschichten angetroffen. Arterien und Venen in den tieferen Schichten bleiben unverändert. Der erwähnte Obliterationsprozeß kann auch nach so leichtem Gefrieren eintreten, daß die Epidermis nicht vollständig zerstört wird, und die Kohlensäure erhält dadurch nach $\mathrm{S}$ alomon eine gewisse elektive Wirkung auf die Gefäße, was eine gute Erklärung für die ausgezeichneten kosmetischen Resultate abgeben könnte, die bei richtiger Dosierung sich durch Kohlensäurebehandlung oberflächlicher Angiome erzielen lassen.

Obliterative Veränderungen in den Gefäßen von der Beschaffenheit, wie Salom on sie geschildert hat, habe ich nirgends angetroffen, und es scheint mir nicht ausgeschlossen, daß seine Auffassung der erwähnten Erscheinungen als Abstoßung und Regeneration von Endothel möglicherweise auf einer irrtümlichen Deutung beruht. Besonders eines der von ihm mitgeteilten 
Bilder (Nr. I) ist geeignet, Zweifel zu erwecken. Die Form der Zellen, ihre zusammenhängende glatte Begrenzungslinie gegen das Lumen und die von dem übrigen Protoplasma abweichende Oberflächenschicht sowie ihre Ablösung in kompaktem Zusammenhang von einer ähnlicher Zellen entbehrenden Wand, also ähnlich einem Schrumpfungsprozeß, alles dies scheint mir eher darauf hinzudeuten, da $\beta$ die Gegenstände, die er beschrieben Schweißdrüsen gewesen sind. Auch Herr Prof. H e d r é n erachtete dies für wahrscheinlich.

Was den schmerz betrifft, den die Behandlung hervorruft, so kann ich bestätigen, daß derselbe im allgemeinen sehr unbedeutend ist; besonders gilt dies für Applikationen, die weniger als I Minute dauern, während längeres Gefrieren stärkeren Schmerz erzeugt, der jedoch binnen kurzer Zeit verschwindet und in hohem Grade durch einen feuchten Umschlag gelindert wird. In Ubereinstimmung mit $\mathrm{H} \mathrm{u} \mathrm{b} \mathrm{b} \mathrm{a} \mathrm{r}$ habe ich starken Schmerz bei Gefrieren im Gesicht auftreten sehen, in einem Falle von Lupus nasi bei I Minute langem Gefrieren der Nasenspitze, in einem anderen Fall bei 2 Minuten langem Gefrieren eines Endothelioms im inneren Augenlidwinkel und bei 3 Minuten langem Gefrieren einer gleichartigen Geschwulst im Nacken; das gleiche war der Fall bei zwei alten Frauen, die lebhafte Zeichen von Schmerz bei mehrminutiger Behandlung in der Schläfen- und Jochbeingegend von sich gaben. Andererseits haben vielmals Patienten bei Behandlung von Gesichtsleiden nichts von Schmerz geäußert; die individuelle Empfindlichkeit spielt sicherlich hierbei eine große Rolle.

\section{Spezielle therapeutische Erfahrungen. 1. Naevi.}

Die flachen Pigment-Naevi, die ich behandelt habe, sind klein gewesen und haben im allgemeinen mittels einoder $z$ weimaligen Gefrierens von $20-30$ Se$\mathrm{k} u \mathrm{n} d \mathrm{e} \mathrm{n}$ entfernt werden können.

Die warzenähnlichen $\mathrm{Naevi}$ haben als wirkliche Geschwulstbildungen eine weit $\mathrm{kräftigere} \mathrm{Behandlung} \mathrm{er-}$ 
fordert und sind erst nach wiederholten Behandlungen während 20-90 Sekunden jeden ro.-I4. Tag verschwunden. Hier ist ja nicht nur eine oberflächliche Blasenbildung und Abstoßung erforderlich, sondern auch Nekrose und Abstoßung oder Resorption des Stromas der Geschwulst. Bei sehr kräftiger Behandlung ließe sich $\mathrm{ja}$ in einer Sitzung die ganze Geschwulst entfernen, dann aber auf Kosten des kosmetischen Resultats, da bei vollständiger Nekrose der ganzen Masse diese abgestoßen werden und Heilung vom Rande aus eintreten muß, während die mikroskopischen Nekrosen bei wiederholten kürzeren Behandlungen allmählich ein Zusammensintern der Geschwulst herbeiführen.

\section{Warzen.}

W a r z e n bieten ein wenn möglich noch dankbareres Feld für die Behandlung mit Kohlensäureschnee; wenigstens gilt dies für die gewöhnlichen e $\mathrm{r} h \mathrm{a} b \mathrm{~b}$ e $\mathrm{n}$ Warzen verschiedenartiger Typen, während die eingedrückten Warzen, vom ,Plantartypus“, nicht gleich günstige Bedingungen darbieten.

Was die gewöhnlichen erhabenen Warzen betrifft, so ist eine einzige Behandlung mit Kohlensäureschnee unter starkem Druck von 30 Sekunden bei kleineren, weicheren Warzen, bis zu 2-3 Minuten bei großen und stark verhornten gewöhnlich genügend, um innerhalb einiger Tage die ganze Warze durch eine $\mathrm{B}$ l a s e n bild u $\mathbf{n} \mathrm{g}$ abzuheben, die, wenn die Behandlung richtig dosiert war, kaum eine sichtbare Narbe hinterläBt. Die rasche Heilung in solchen Fällen scheint mir darauf hinzudeuten, daß die Epithelisierung von tiefliegenden, nicht nekrotisierten Basalzellen innerhalb der Basis der Warze ausgeht. Die Abwesenheit von Rezidiven in den Fällen, die ich längere Zeit (ro bis II Monate) hindurch habe verfolgen können, spricht dafür, daß der Infektionsstoff, der erwiesenermaßen die Warzen hervorruft, durch das Gefrieren zerstört wird, was entweder so vor sich gehend gedacht werden kann, daß er in den Hornmassen lokalisiert ist und mit diesen entfernt wird, oder daß er seine Lebensbedingungen bei der Entzündung verliert, die durch die Behandlung hervorgerufen wird. Eine direkte Zerstörung des Ansteckungsstoffes während der Behandlung dürfte indessen nicht stattfinden; da- 
gegen sprechen die negativen Resultate, die man bei Einwirk ung hoher Kälte auf Mikroorganismen erhalten hat.

Eine exakte Dosierung wird am besten dadurch erreicht, daß man einen der Größe und Form der Warze wohlangepaßten Stab verwendet und das Gefrieren so lange fortsetzt, bis ein kleiner, schmaler, weißer Rand um die Warze herum auftritt, ein Zeichen dafür, daß die Warze selbst bis zur umgebenden Haut hin durchfroren worden ist.

Bei der Behandlung kann man die Blase und die mit derselben abgehobene Warze (siehe Fig. 2) wegschneiden und danach die entstandene nässende Fläche mit einem kleinen Verbande bedecken, praktischer aber ist es, ganz einfach die Blase ein-

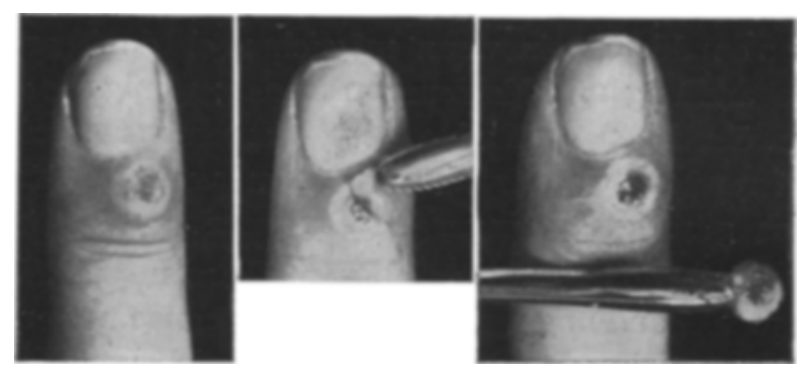

Fig. 2.

trocknen zu lassen und erst nach 8-io Tagen die nunmehr abgelöste Warze zu entfernen oder sie von selbst abfallen zu lassen, wobei ein Verband niemals angebracht zu werden braucht. Eine Reimplantation der Warze nach dem Eintrocknen der Blase kommt nicht zustande. Fig. 3-5 veranschaulichen den Effekt der Behandlung.

Die stark verhornten Warzen (Fig. 3) wurden am 27. V. 60 Sekun den lang ohne Resultat behandelt. Ein Teil der Hornschicht wurde weggeschnitten und die Stellen am 29. V. einem 2 Minuten langen Gefrieren unterzogen. Am Io. VI, waren die Warzen abgefallen, an ihrer Stelle war aber die Epidermis noch stark verdickt (Fig. 4), weshalb eine weitere Behandlung von 60 Sekunden ,,der Sicherheit wegen" ausgeführt wurde. Als Pat. sich Ende Oktober vorstellte, fanden sich dort, wo die Warzen gesessen hatten, nur einige kleine, kaum sichtbare Narben (Fig. 5). 
Warzen vom $\mathrm{Plantartypus}$ bestehen aus einem in die Haut eingedrückten konischen Zapfen von verhornter Epidermis, dessen Basis nicht oder nur unbedeutend sich über die oft schwielig verdickte Haut der Umgebung erhebt und dessen Spitze mehr oder weniger tief, bis $\mathrm{zu} 8 \mathrm{~mm}$ in einem meiner Fälle, in das Corium eingesenkt ist. Daß diese Warzen der Behandlung

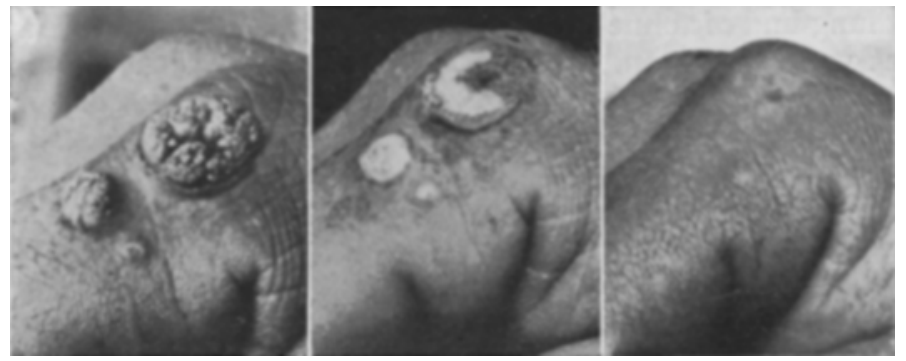

Fig. 3.

Fig. 4.

Fig. 5.

schwerer zugänglich sein müssen als die des gewöhnlichen erhabenen Typus, geht bereits aus dem hervor, was oben betreffs der Tiefenwirkung des Kohlensäuregefrierens angeführt worden ist. Der Unterschied läßt sich am einfachsten durch nachstehende
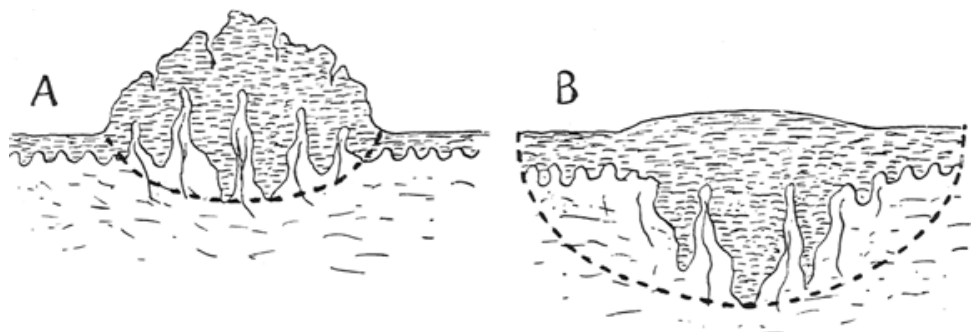

Fig. 6.

Figur 6 veranschaulichen, welche schematische Querschnitte a) einer gewöhnlichen erhabenen Warze und b) einer „Plantar“Warze zeigt und die Grenzen des für eine radikale Wirkung nötigen Gefrierens durch die punktierte Linie angibt.

Beim ersteren Typus kann man mit einem Gefrieren, das nicht weiter als bis zum Rande der Warze bemessen wird, unter die Epidermisschicht hinabgelangen und demnach die Abspren- 
gung der hypertrophischen Epidermispartien bis zu gewünschter Tiefe dosieren, ohne die Umgebung zu schädigen. Beim letzteren Typus dagegen liegen die basalen Hornschichten in oft großer Ausdehnung so tief, daß das Gefrieren sie nicht erreicht, wenn man nicht eine größere Oberfläche als die, welche von der Warze selbst eingenommen wird, durchfriert.

$\mathrm{Da} B$ indessen auch Warzen vom Plantartypus durch energischere Behandlung und oft ohne größere Unzuträglichkeiten für den Patienten entfernt werden können, ist sicher. So habe ich z. B. an mir selbst mittelst Gefrieren während 3 Minuten eine hartnäckige, ,eingedruickte“ Warze auf der Daumenkuppe beseitigt.

Bei einem Fall von Warzen in der Planta pedis, die ich mehrere Male je 30 Sekunden lang behandelte, hatte dies keinerlei Wirkung; in einem zweiten Falle blieb ein Gefrieren während 2 Minuten, in einem dritten Falle zweimaliges Gefrieren von bzw. 3 und $3^{1 / 2}$ Minuten resultatlos. Eine derartige Warze auf der Volarseite der Basis des Daumens verschwand dagegen nach 2 Sitzungen mit 45 bzw. 60 (?) Sekunden langem Gefrieren. Andere Fälle von „Plantar"-Warzen habe ich nicht behandelt.

Auch gegen Keloide ist Kohlensäurebehandlung zur Anwendung gekommen, und zwar nach der Angabe einiger Antoren mit gutem Resultat. Ich selbst habe ein bohnengroßes Keloid behandelt und durch zweimaliges Gefrieren von je 6o Sekunden eine bedeutende Verminderung der Geschwulst erreicht; die Behandlung wird fortgesetzt.

\section{Angiome.}

Ich habe Gelegenheit gehabt, ungefähr 40 Fälle von Angiom zu behandeln, darunter 6 Fälle von flachen Feuermälern, etwa 20 typische, mehr oder weniger hypertrophische Angiome bei kleinen Kindern sowie einige Fälle von kleineren kavernösen Angiomen oder Ansammlungen von Teleangiektasien.

Meine Erfahrungen betreffs der Behandlung von Angiomen mit Kohlensäure in fester Form kann ich folgendermaßen zusammenfassen: Die ausgebreiteten, typischen planen Angiome eignen sich schlecht für diese Behandlungsmethode wie auch für sonstige bisher geprüfte Methoden -; höchstens wird durch langdauernde Behandlung einige Besserung erreicht. 
Um so dankbarer sind im allgemeinen die bei kleinen Kindern gewöhnlichen, mehr oder weniger hypertrophischen (über die umgebende Haut erhabenen) Angiome, die sich nicht tiefer alshöchstens einige Millimeter unter das $\mathrm{Niveauder} \mathrm{Haut}$ erstrecken. Diese können durch einige wenige oder mehrere Behandlungen

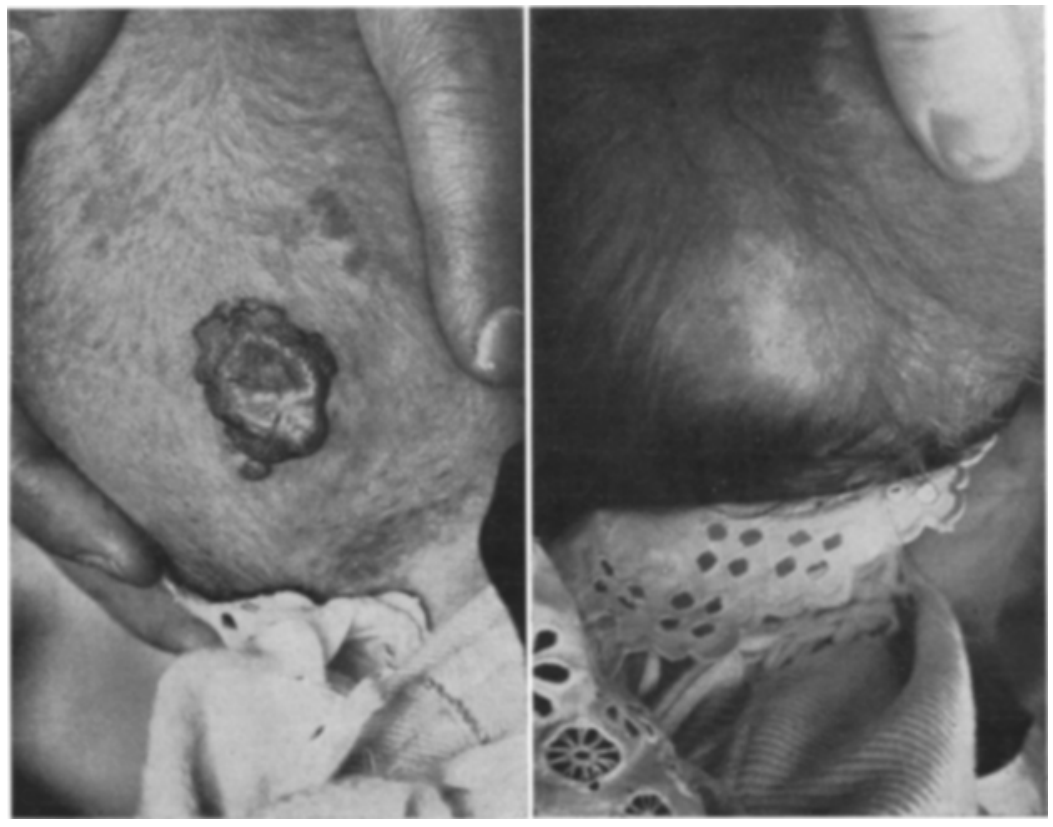

Fig. 7. 6 Wochen altes Mädchen. Mark- Fig. 8. Behandlung 4 Mal 1o-2o Sek. großes etwas erhabenes, teilweise ulceriertes Angiom der Nackenhaut.

während 2 Monaten. Nach b ist das Angiom verschwunden.

von I0-20 Sekunden, die in Zwischenräumen von einigen Wochen wiederholt werden, zu fast spurlosem Verschwinden gebracht werden (Fig. 7 und 8). J e na ch der T i efe des Ang io ms gibt die Behandlung gröBere odergeringere A ussicht auf eine vollständige Beseitigung der Geschwulst - sofern nicht das Gefrieren bis zu vollständiger Nekrose der ganzen Partie getrieben wird. Günstig für Kohlensäurebehandlung sind auch die kleinen, oberflächlichen, 
diffus begrenzten, höchstens erbsengroßen Flecke, die auf mikroskopischen Teleangiektasien beruhen, sowie $\mathrm{kle}$ in e re kavernöse Angiome. Bezüglich der ausgebreiteteren kavernösen Angiome fehlt es mir an Erfahrung.

Wie die Heilung des Angioms zustandekommt, scheint mir nicht völlig klar. Da die zurückbleibenden Narben nach gelungenen Behandlungen äußerst unbedeutend, oberflächlich, oft kaum wahrnehmbar sind, so ist es ganz sicher, daß eine totale, sich durch die ganze Tiefe des Angioms erstreckende Nekrose nicht hervorgerufen zu werden braucht, um die überflüssigen Gefäßmassen zum Verschwinden zu bringen und somit eine elektive Wirkung des Gefrierens auf die Gefäße zu erhalten. Es liegt da am nächsten, die Heilung durch eine Obliteration infolge von Thrombenbildung zu erklären, und daß eine solche wirklich infolge des Gefrierens ohne Nekrose umgebenden Gewebes entstehen kann, habe ich oben gezeigt, gleichzeitig aber darauf hingewiesen, daß sie bei den kurzdauernden Applikationen, um die es sich hier handelt, keineswegs besonders verbreitet $\mathrm{zu}$ sein scheint. Salomons Erklärung: Obliteration der Gefäße durch Ausfüllung mit abgestoßenem und wieder wachsendem Endothel beruht, wie erwähnt, möglicherweise auf einer Fehldeutung. Daß makroskopisch sichtbare Teleangiektasien, auch wenn sie ganz an der Hautoberfläche liegen, nach einem 30 Sekunden langen Gefrieren dem Anschein nach unbeschädigt bleiben und ebenso frei wie vorher den Blutstrom durchlassen können, habe ich Gelegenheit gehabt, bei der Behandlung eines Falles von Lupus nasi bei einer erwachsenen Frau zu beobachten. Eine andere Erklärung für das Verschwinden des Angioms als Thrombenbildung kann ich jedoch nicht finden. Freilich bleibt dann das eigentümliche Verhältnis bestehen, daß die planen Angiome, die aus mikroskopischen oder für das unbewaffnete Auge eben sichtbaren Teleangiektasien bestehen, nach mehrmonatigen wiederholten, energischen Behandlungen kaum irgendwelche Veränderungen aufweisen, während kleine, mehr diffuse Angiomflecke, aus dem Anschein nach vollständig gleichen Angiektasien zusammengesetzt, nach einer einzigen Behandlung verschwinden. $\mathrm{Daß}$ die Angiome bei sehr kleinen Kindern oft so leicht zum Verschwinden gebracht werden können, könnte vielleicht auf 
einem besonders hohen Grade von Empfindlichkeit bei den Gefäßwänden mit dadurch leichter eintretender Thrombenbildung und Obliteration beruhen.

\section{Hautkrebs.}

Gefrieren mittelst Kohlensäureschnee ist mehrfach mit Erfolg als Heilmittel gegen Hautkrebs angewandt worden. Schon a priori scheint die Methode sich hierzu zu eignen; bei hinreichend kräftiger Applikation wirkt ja der Kohlensäureschnee als ein zerstörendes Kaustikum, wobei er vor anderen solchen den Vorteil hat, relativ schmerzfrei zu sein und sehr gut dosiert werden zu können. Die Erfahrungen von verschiedenen Seiten her sind auch ziemlich einstimmig betreffs der guten Wirkung des Gefrierens bei oberflächlichem Hautkrebs. Daß in die Tiefe gehende Krebsvegetationen nicht würden beeinflußt werden können, ist im Hinblick auf das, was oben betreffs der geringen Tiefe, bis zu welcher das Gefrieren vordringen kann, gesagt worden ist, selbstverständlich.

In der mir zugänglichen Literatur sind indessen die Angaben über die Behandlungsresultate bei Hautkrebs ziemlich spärlich.

Pusey selbst hat Kohlensäure bei ,epitheliomatous degeneration“ seniler Keratosen angewandt, scheint aber der Methode kein Vertrauen auBer bei sehr kleinen und sehr oberflächlichen Formen von Krebs entgegenzubringen.

Zeisler hat I3 Fälle behandelt. Er wandte eine Applikationszeit von 60 Sek. und starken Druck an und hat ideale Heilungen in fast allen Fällen, bisweilen nach einer einzigen Sitzung erhalten. In einigen wenigen der Fälle ist Röntgenbehandlung nach dem Gefrieren zur Anwendung gekommen. Der älteste der mit Kohlensäure behandelten Fälle, geheilt nach einem einzigen Gefrieren, ist 6 Monate hindurch verfolgt worden, ohne Zeichen von Rezidiv zu zeigen.

Jackson und Hubbard teilen ohne nähere Angaben über die Fälle mit, daß Hautkrebs vom Typus des Ulcus rodens ihrer Erfahrung nach eine sehr geeignetes Objekt für Gefrierbehandlung bilden, die durch ihre Raschheit und Schmerzlosigkeit andere kaustische Methoden übertrifft. Die Applikationszeit beträgt je nach der Dicke der Geschwulst 30-90 Sek., bei kräftigem Druck. Von besonderem Interesse ist die Angabe der Verfasser, da $\beta$ sie behandelte Fälle mehrere Jahre verfolgt haben, ohne ein Rezidiv zu sehen, daß in anderen Fällen Rezidiv eingetreten, dieses aber mit größter Leichtigkeit durch eine neue Behandlung behoben worden sei. 
Sutton erwähnt ganz summarisch, daß er ideale Heilung von Hautkrebs durch Gefrieren mit Kohlensäure während 40-45 Sek. mit erneutem Gefrieren während 30 Sek. oder länger nach dem Auftauen erhalten habe.

Sauerbruch, Zweig und Macleod teilen einige mit ausgezeichnetem unmittelbarem Resultat bəhandelte Fälle mit. Gottheil erwähnt einen Fall von Ulcus rodens der Unterlippe, der nach erfolgloser Röntgenbehandlung durch ein Gefrieren mittels Kohlensäure während 60 Sek. zur Heilung gebracht wurde; die Ränder der Narbe waren jedoch noch verdächtig. Nobl und Springels haben die Methode in 9 Fällen von Epitheliom angewandt. 3 Fälle waren zur Zeit der Veroffentlichung noch unter Behandlung, in guter Heilung begriffen. Von den übrigen hatte I nach Heilung des Geschwürs eine andere Behandlung wegen fortschreitender Infiltration am Rande erforderlich gemacht; die anderen 5 waren vollständig geheilt, darunter ein mehr als kinderhandgroßes Epitheliom. Einer von den Fällen war nach beendeter Behandlung mehrere Monate hindurch verfolgt worden, ohne Rezidiv zu zeigen.

Nach einer Angabe in Puseys Arbeit vom Jahre Igro sollen außer diesen auch Schaleck und Foerster mit Erfolg die Methode angewandt haben.

Von großem Interesse sind die Bemerkungen über den Wert der Kohlensäuremethode im Vergleich mit der Röntgenbehandlung, die sich bei einigen der genannten Autoren finden.

Zeisler stellt die Gefriermethode in kosmetischer Hinsicht der Röntgenmethode gleich. Nach Sutton sind die kosmetischen Resultate fast ebensogut wie bei Röntgenbehandlung, vor welcher seine Ansicht nach die Kohlensäuremethode wegen ihrer raschen Wirkung, ihrer Einfachheit und Billigkeit den Vorzug besitzt. Foerster und Jackson-Hubbard halten die Kohlensäuremethode für besser als die Röntgenmethode.

Sämtliche Autoren scheinen clarin einig zu sein, daß nur oberflächliche Formen von Hautkrebs einer Behandlung mit Kohlensäureschnee zu unterziehen sind, daß die Resultate hier aber auch ausgezeichnet sind.

Pusey betont, daß er bei größeren und tiefergehenden Veränderungen entschieden Röntgen vorzieht, sowie daß solche Fälle, wo der Krebs bereits in das subkutane Gewebe hinabgedrungen ist, seiner Ansicht nach mit größerem Erfolge mit Curettement und Kauterisation mit Zinkchlorid oder kaustischem Kali oder mit Röntgen zu behandeln sind.

Einen Vergleich mit den Resultaten der Radiumbehandlung habe ich in der Literatur nicht angetroffen. 
Die Kohlensäurebehandlung bei Hautkrebs ist dem Anschein nach nirgends zum Gegenstand einer eingehenderen Darstellung gemacht worden, und da diese Frage nicht geringe Bedeutung und Interesse besitzt, so habe ich es für angezeigt gehalten, ausführlicher über die Resultate $\mathrm{zu}$ berichten, die mein eigenes Material aufzuweisen hat.

Dasselbe umfaßt folgende Fälle.

Nr. 1. Anna Sofia J., 78 J. Stockh. Offentl. Versorgungsanstalt, Westpavillon, I9Io, Nr. III7.

Ulcera rodentia faciei. Path.-anat. Diagnose: Kankroid. A) Ein zweimarkstückgroßes Geschwür gleich unterhalb des inneren Augenwinkels auf der linken Seite, mit runder Kontur, ebenen Rändern und einige Millimeter tief eingesenktem, glattem, blaßgelbrotem, nässendem Grunde. B) Ein gut erbsengroßes, rundes Geschwür auf der Nasenwurzel mit kaum eingesenktem, nässendem, hellrotem Grunde.

I2. XII. Behandlung jedes Geschwürs während 30 Sek. Am folgenden Tage Odem auf dem Geschwürsgrunde und in dessen nächster Umgebung; danach schienen die Geschwüre sich zu reinigen; erweiterte Gefäße in der Umgebung des kleinen Geschwürs sichtbar, im übrigen keine Veränderung bis zum 3r. XII., wo Pat. infolge einer ausgebreiteten Gangrän des einen Beines starb. Aus jedem der beiden Geschwüre wurde bei der Sektion ein Stück behufs pathol.-anat. Untersuchung excidiert, und diese ergab folgendes Resultat. A) Das große Geschwür: das Präparat zeigt das Bild eines typischen Kankroids. B) Das kleine Geschwür: das Präparat zeigt nur eine geringe Anzahl Kankroidperlen. Die strangförmige Proliferation wird charakterisiert durch schmale Epithelstränge mit einer geringeren Anzahl Zellen in jedem Strange. Das Protoplasma der Zellen erscheint geringer. Die Zellen selbst erscheinen daher dichter als im vorhergehenden Präparat zu liegen. Das mikroskopische Bild erinnert an einen Scirrhus.

In diesem Falle besaß ich noch keine Erfahrung betreffs der Behandlung, begann dieselbe daher sehr vorsichtig mit nur 30 Sekunden, und wartete das Resultat der ersten Behandlung ab, die auch infolge des Todesfalles die einzige blieb. Die mikroskopische Untersuchung bestätigte die Diagnose und zeigte, daß die Behandlung unzureichend gewesen war, um die Krebselemente zu zerstören.

Nr. 2. August A., 35 J., Bauer. Serafimer-Lazarett, chirurg. Poliklinik, IgII, Nr. 860.

Ulcus rodens labii sup. Pathol.-anat. Diagnose: Plattenepithelkrebs. 
Seit 3 Jahren ein gut erbsengroßes, oberflächliches Geschwür auf der Oberlippe dicht unterhalb der Wurzel des rechten Nasenflügels. Das Geschwür ist mit einer dünnen Kruste belegt, bei deren Entfernung ein leicht blutender, ebener Geschwürsgrund zutage tritt.

25. I. IgII. Behandlung während 30 Sek. unter kräftigem Druck. 26. I. Das ganze Geschwür etwas angeschwollen, hellrot, unbedeutend erhaben, einer mukösen Papel ähnelnd. Erneute Behandlung während 20 Sek. 2. II. Das ganze Geschwür mit einer dicken Kruste belegt, bei deren Ablösung sich ein ebener Geschwürsgrund darbietet, umgeben von einem kleinen hyperämischen Walle. Das Geschwür sieht rein aus und scheint in Heilung begriffen zu sein. Leichte Lapisierung wegen Blutens. 4. II. Die Heilung schreitet deutlich vom Rande aus fort; das Geschwür nur weniger als erbsengroß, sieht gesund aus, umgeben von einem hellroten, schwach erhabenen Wall; dicht am Nasenflügel eine kleine Fissur. I8. II. Das Geschwür vollständig geheilt, mit Epidermis von derselben Farbe wie die Umgebung, ohne deutliche Narbenbildung. Der Rand des Geschwürs noch angedeutet durch einen schwachen Wall von unebenem, etwas warzenähnlichem Aussehen. Bei Versuchen, die äußerst kleinen Hornexkreszenzen wegzunehmen, entsteht Blutung. Der geheilte Geschwürsgrund fühlt sich normal an, nicht infiltriert. 3. VIII. Andauernd geheilt mit unsichtbarer Narbe. Die Haut an der fraglichen Stelle glatt, weich und von derselben Farbe wie die Umgebung.

28. X. IgII und 20. III. Igr2. Laut brieflicher Mitteilung andauernd geheilt.

Also ein kleiner, sehr oberflächlicher Hautkrebs, der nach zwei mit eintägiger $Z$ wischenzeit ausgeführten Behandlungen von insgesamt 50 Sekunden binnen 25 Tagen heilte. Die Heilung bei Nachuntersuchung $1 / 2$ Jahr nach der Behandlung noch andauernd, in kosmetischer Hinsicht ideal, und laut brieflicher Mitteilung nach weiteren 7 Monaten ohne Veränderungen bestehend.

Nr. 3. Gustav L., 63 J., Pächter. Serafimerlazarett, chirurg. Poliklinik, I9II, Nr. I352.

Cancer cutis reg. infratemporalis. Pathol.-anat. Diagnose: Plattenepithelkrebs.

Seit 6-7 Jahren ein Geschwür in der rechten Schläfengegend an der Haargrenze dicht vor dem Ohr, anfangs sehr klein, allmählich anwachsend. Jetzt ein nahezu markstückgroßes, kraterförmiges Geschwür mit aufgetriebenem Rande, auf das äußere Ohr übergreifend. Gehörgang normal. Regionäre Lymphdrüsen nicht angeschwollen.

7. II. Behandlung mittelst eines Kohlensäurestabes, der sukzessiv gegen die verschiedenen Teile des Geschwürs und die Haut in der nächsten 
Umgebung während I Minute (3o Sek. ?) gedrückt wurde. Die Behandlung wurde am folgenden Tage wiederholt.

24. II. Der Geschwürsgrund zum größten Teile von gesundem Aussehen mit hellen, reinen Granulationen. In einem excidierten Stücke des Geschwürsgrundes sieht man mikroskopisch Kolben von Plattenepithel, ziemlich vereinzelt und von jungem, zellreichem Bindegewebe umgeben. Das Gewebe in seiner Gesamtheit reich von Rundzellen infiltriert. Erneute Behandlung 30 Sek., eine Partie des Geschwürs 60 Sek. Die Behandlung wurde am folgenden Tage wiederholt.

I4. III. Das Geschwür sieht bedeutend reiner aus. Die Partie dicht am Ohre geheilt, der obere Rand noch unterminiert, fühlt sich infiltriert an; der untere Rand in guter Heilung begriffen; der Grund

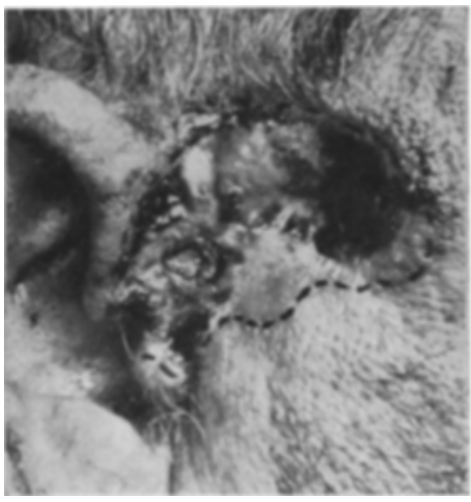

Fig. 9. Die punktierte Linie bezeichnet die ursprüngliche Grenze des Geschwüre. 6. IV.

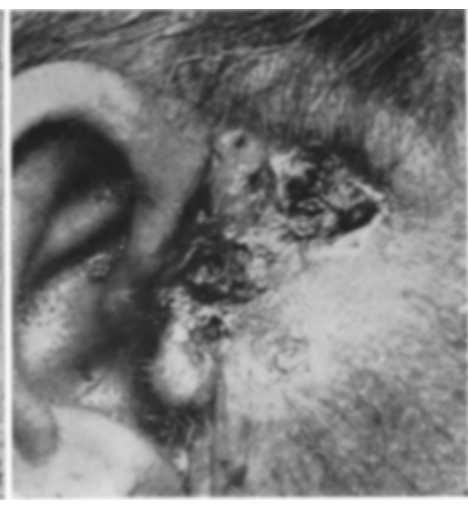

Fig. Io wie 5. V.

uneben, teilweise üppig granulierend, teilweise tief eingesenkt, unrein, fühlt sich fest und infiltriert an. Die mikroskopische Untersuchung zeigt andauernd Plattenepithelkrebs. Der obere infiltrierte Rand des Geschwürs wurde nun I Minute lang behandelt. Die Granulationen wurden weggekratzt, und das Geschwür lapisiert. Nach I2 Tagen erneute Behandlung, diesmal 2 Min. auf jedem von 5, den oberen Geschwürsrand, den oberen Teil des Geschwürsgrundes und die angrenzende Haut umgebenden Feldern.

6. IV. In guter Heilung begriffen (siehe Fig. 9). Der vordere und der obere Rand wurden 3 Minuten lang behandelt; einige erbsengroße Geschwüre innerhalb der bereits geheilten Partie I Minute lang. Lapisierung diєser kleineren Geschwüre und eines Teiles des großen Geschwürs.

5. V. Die Heilung hat weitere Fortschritte gemacht (siehe Fig. I0); 
der größte Teil des Geschwürs ist nun geheilt und die Narbe ist von einer feinen und ebenen Beschaffenheit. Erneute Behandlung (Zeitdauer nicht vermerkt, wahrscheinlich 3 Min.).

7. VI. Die vorderen zwei Drittel des Geschwürs nahezu geheilt; längs dem vorderen Ansatz der Ohrmuschel noch ein mandelgroßes Geschwür. Behandlung des letzteren Geschwürs während 2 Min., wiederholt nach 2 Tagen, eines kleinen oberflächlichen Geschwürs innerhalb des vorderen Teils der Narbe während 40 Sek.

Pat. war seitdem verhindert, sich zur Behandlung einzufinden; er schreibt am I5. IX., daß das Geschwür ,,ruhig und still ist, aber wohl etwas mehr Behandlung erfordert".

28. III. I9I2. Das seit 9 Monaten nicht behandelte Geschwür ist wieder größer und tiefer geworden; wirł mit Kadium behandelt werden.

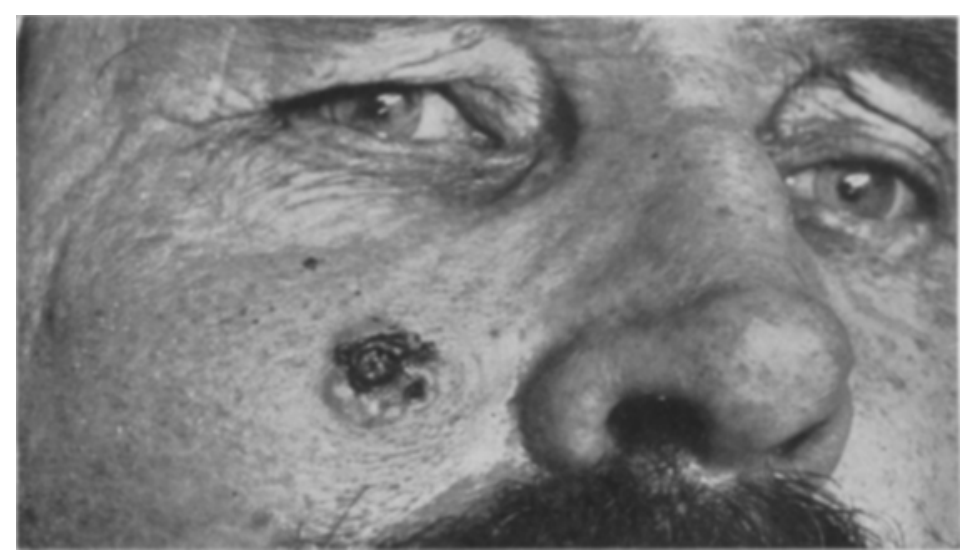

Fig. I I.

In diesem Falle hatte der Krebs bereits ein größeres kraterförmiges Geschwür gebildet, das, seiner Tiefe nach zu urteilen, vollständig die Haut und sicherlich außerdem einen Teil des Unterhautgewebes durchfressen hatte; die Ränder des Geschwürs waren geschwulstartig aufgetrieben. Die Behandlung wurde so angeordnet, daß der Patient, der auf dem Lande wohnte, zunächst einmal ungefähr alle drei Wochen, dann einmal im Monat nach Stockholm kam, wobei das Geschwür energisch mit Gefrieren zuerst $1 / 2$ - I, dann bis zu 3 Minuten jedesmal behandelt wurde, was bei einigen Versuchen nach I oder 2 Tagen wiederholt wurde. Im Laufe von 4 Monaten, während welcher der Patient das Krankenhaus sechsmal besucht und Io Behandlungen erhalten hatte, 
trat eine zwar langsame, aber stetig fortschreitende Heilung mit ungewöhnlich schöner Narbenbildung ein. Leider wurde Pat., ehe noch vollständige Heilung erreicht worden war, daran verhindert, vorläufig die Behandlung fortsetzen zu lassen.

Nr. 4. A. G. L., 62 J., Steinsetzer. Serafimer-Lazarett, chir. Abt. II, IgII, Nr. 285.

Cancer cutis reg. infraorbitalis dextr. Pathol.-anat. Diagnose: Plattenepithelkrebs.

Seit 8-9 Jahren auf der rechten Wange ein pfennigstückgroßes, zeitweise geheiltes, nie schmerzendes Geschwür. Das Aussehen desselben vor der Behandlung geht aus Fig. II hervor. Die Ränder des Geschwürs

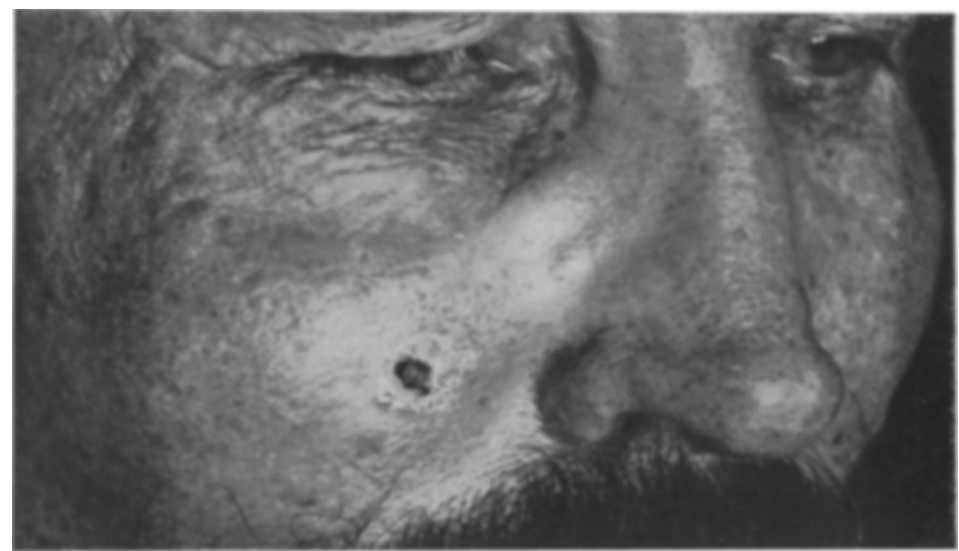

Fig. I2.

sind teilweise wallförmig aufgetrieben, von fester Konsistenz, bedeckt mit Haut von normaler Farbe.

I3. IV. Behandlung 3 Min. in einer Sitzung unter kräftigem Druck. In den nächsten Tagen danach trat eine beträchtliche Schwellung und Rötung der rechten Gesichtshälfte ein; das rechte Auge schwoll zu; kein Fieber.

29. IV. Das Geschwür geheilt; dessen Mitte noch mit einer kleinen, lose sitzenden Kruste bedeckt (siehe Fig. I2). Im unteren Teil der Narbe noch einige niedrige Knoten, die auf dem Bilde andeutungsweise zu sehen sind. Bei Druck mittelst einer Glaslinse zeigt die ganze Narbe eine eigentümliche Zeichnung von dichtstehenden, feinen, weißen Punkten, aller Wahrscheinlichkeit nach unter der neugebildeten Epidermis noch zurückgebliebenen Hornperlen. Erneute Behandlung 30 Sek. 
4. VIII. Vollständig geheilt mit weicher Haut von normaler Farbe; die Narbe so gut wie unsichtbar, unterscheidet sich nur durch ihre etwas glattere Oberfläche von der umgebenden Haut (siehe Fig. I3).

3I. X. IgII, 4. IV. und 30. VI. IgI2. Andauernd geheilt.

Eine einzige kräftige Behandlung (3 Minuten) führte also in diesem Falle Heilung innerhalb I6 Tagen herbei; die Narbe wurde der Sicherheit halber noch weiter eine $1 / 2$ Minute behandelt. Die Heilung besteht noch nach I4 Monaten fort und ist in kosmetischer Hinsicht ideal.

Nr. 5. Sofia E., 72 J., Witwe. Serafimer-Lazarett, chir. Poliklinik, IgII, Nr. 4366.

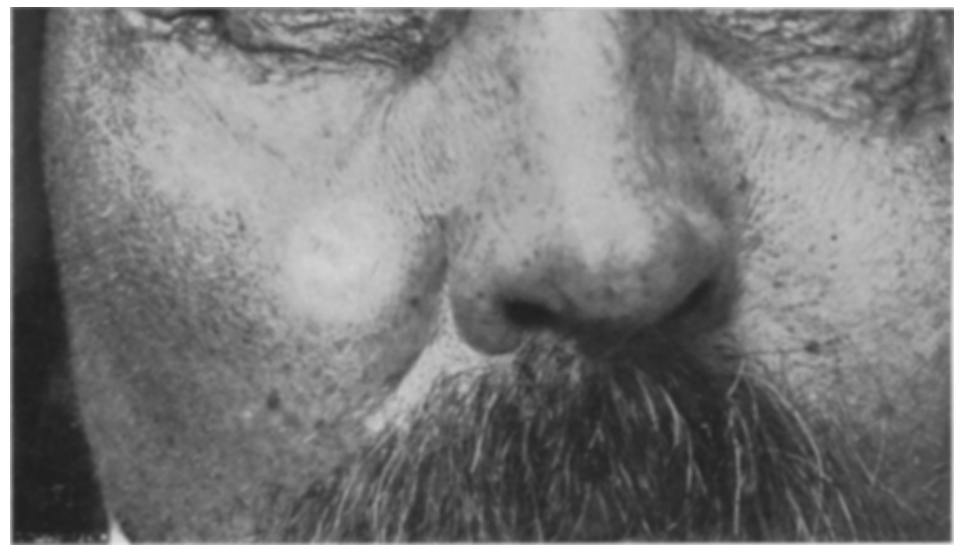

Fig. I3.

Cancer cutis reg. temporalis dextr. Pathol.-anat. Diagnose: $\mathrm{Kan}$ kroid.

Von einer Brandverletzung im Alter von 3 Jahren hat Pat. eine über die beiden Seiten des Gesichts und der Stirn gehende Narbe, teilweise stark pigmentiert, ohne nennenswerte Schrumpfungen. Nach Ablösung einer kleinen Hautschuppe in der rechten Schläfengegend entstand Weihnachten rgro ein Geschwür, das seitdem unablässig an Größe zugenommen hat. Es ist nun eine zweimarkstückgroße, breiige, stinkende Ulceration mit wallartigen Rändern und einem Knoten von der Größe einer Kleinfingerkuppe und von typischem Kankroidaussehen im oberen Teile. Der Geschwürsgrund läßt sich gegen den darunterliegenden Knochen verschieben.

Die Behandlung wurde mit I Minute dauernder Applikation am 29. IV. beg onnen, danach mit Sitzungen von $\mathrm{I}-2$ Minuten am 2., 6 , 9., I6., 23. und 30. Mai fortgesetzt. Hierbei wurde das Geschwür reiner, 
die hypertrophischen Partien sanken auf das Niveau der Umgebung hinab, und Heilung begann vom Rande aus einzutreten, so daß am 30. Mai große Teile der vorderen Hälfte des Geschwürs epidermisiert waren. Am 30. VI. wurde erneute Behandlung während nicht weniger als 5 Minuten an dem ganzen noch vorhandenen Geschwür vorgenommen, außer dem vorderen Rande, der 3 Min. lang behandelt wurde, und einer kleinen, isolierten Ulceration innerhalb des bereits geheilten Gebietes, die I Min. lang behandelt wurde. Pat. klagte über starken Schmerz bei der Behandlung, was wohl der Anlaß war, weshalb sie danach bis 26 . VII. wegblieb.

Die Heilung war währenddessen nicht fortgeschritten; eher war das Geschwür größer geworden und war nun von über die Ränder erhabenen, festen, kankroidähnlichen Massen angefüllt. Die mikroskopische Untersuchung derselben ergab Kankroid. Nun wurde alles weggekratzt, was mit dem scharfen Löffel zu entfernen war, wobei der Bindegewebsgrund unter den Krebsmassen sich als auffallend eben und fest erwies, so daB man den Eindruck einer guten Begrenzung des Krebses nach der Tiefe zu erhielt. Danach wurde das Geschwür mit Lapisstift geätzt. Eine neue Kohlensäurebehandlung wurde diesmal nicht vorgenommen.

Nach 2 Wochen, am 9. VIII., war das Geschwür in erstaunlich rascher Heilung begriffen, nur pfennigstückgroß, bedeckt mit einer dünnen, gelben Kruste und umgeben von einem $5 \mathrm{~mm}$ breiten, neugebildeten Epidermisrande.

I4. VIII. Zwei typische, ungefähr erbsengroße Kankroidperlen sind in das Geschwür hinaufgedrungen, das im übrigen mit reinen Granulationen bedeckt ist. Das ganze Geschwür wurde aufs neue gekratzt, und die Teile, wo die Krebsknoten gesessen hatten, wurden mit Kohlensäure 2 Min. lang behandelt, während der Rest des Geschwürs lapisiert wurde.

4. IX. Das Geschwür nun vollständig geheilt; neben dem vorderen Rande desselben aber, nach vorn von dem ursprünglichen Geschwür, ist innerhalb des subkutanen Gewebes ein atheromähnlicher, ungefähr pfennigstückgroßer, flacher Knoten entstanden; der Knoten ist etwas gegen die Unterlage, in geringem Grade aber auch gegen die Haut verschiebbar, von fester Konsistenz, etwas empfindlich. II. IX. Das Geschwür andauernd geheilt. Der erwähnte subkutane Knoten wird excidiert und untersucht: Plattenepithelkrebs. Die Wunde wird offen gelassen. 5. XI. Vollständig geheilt; in der Narbe nach dem vor 2 Monaten excidierten Knoten findet sich eine Verdickung mit etwas unebener und schorfiger Haut; die ganze übrige Narbe glatt, weich, in gleichem Niveau mit der Umgebung und fast von derselben Farbe wie diese.

In diesem Falle hatte die Kohlensäurebehandlung keineswegs 
einen so augenfällig günstigen Effekt wie in dem vorigen, was ja auch wegen der tieferen Ausbreitung des Krebses zu erwarten war. 7 kräftige Behandlungen im Laufe von etwas mehr als einem Monat bewirkten zwar einen deutlichen Rückgang der bösartigen Neubildung und eine partielle Heilung des Geschwürs, trotzdem das Geschwür aber einem weiteren Gefrieren während nicht weniger als 5 Minuten in einer Sitzung unterzogen wurde, waren immer noch lebenskräftige Krebselemente zurückgeblieben, so daß nach einer längeren Zwischenzeit das Geschwür sich wieder von Cancroidmassen erfüllt und deutlich im Wachstum begriffen zeigte. Als Vorbereitung zu einer erneuten Kohlensäurebehandlung wurde das ganze Geschwür ausgekratzt und mit Lapisstift geätzt. Dabei wurde beobachtet, daß das Bindegewebe unter dem Granulationsgewebe und den Krebsmassen einen glatten, festen Grund bildete, der die nach außen davorliegenden, lockeren Gewebe gleichsam abkapselte. Das einfache Abkratzen und die Lapisierung hatte indessen ohne erneuten Gefrieren einen bemerkenswerten Effekt, indem das Geschwür danach erstaunlich rasch zu heilen begann. Einige neue Krebsknoten sprossen aus dem Geschwürsgrunde auf; diese wurden gekratzt und mit Kohlensäure behandelt und danach trat vollständige Heilung ein. Unterdessen begann ein subkutaner Ausläufer des Krebses, außerhalb der Grenze des ursprünglichen Geschwürs, sich zu erkennen zu geben; er wurde exstirpiert, worauf vollständige Heilung eintrat.

Ein gutes Resultat wurde also nach vieler und langwieriger Arbeit erreicht, welchen Anteil aber die Kohlensäurebehandlung an demselben hat, ist schwer $z \mathbf{u}$ entscheiden. Ein glänzendes Resultat ist es jedenfalls nicht, da zunächst $I^{1} / 2$ Monate dauernde energische Behandlung mit nur partiellem Erfolge, was die direkte Zerstörung der Krebsmassen betrifft, gekrönt wurde. Dagegen ist es denkbar, da $\beta$ es die wiederholten Behandlungen gewesen sind, denen zufolge das Bindegewebe unter dem Geschwür eine immer festere und dichtere Beschaffenheit annahm, dadurch allmählich die tiefliegenden Krebsausläufer erstickte und die oberflächlicheren gleichsam abkapselte, was die ungewöhnlich rasche Heilung nach einem einfachen Kratzen und Lapisieren würde erklären können. $\mathrm{Daß}$ eine solche Sklerose um Krebszüge 
herum den Untergang der letzteren herbeiführen kann, ist von $\mathrm{Handley}$ in seiner Arbeit über die Ausbreitungsweise des Brustkrebses ${ }^{1}$ ) nachgewiesen worden.

Der während der Heilung des Geschwürs nach vorn von demselben auftretende subkutane Knoten nahm einen Platz ein, der entweder gar nicht oder nur unbedeutend einer Behandlung unterzogen worden war.

Nr. 6. Edla J., 49 J., Landwirtsehefrau. Serafimer-Lazarett, chir. Poliklinik, I9II, Nr. 4876 .

Ulcus rodens nasi. Pathol.-anat. Diagnose: Krebs.

Seit dem Alter von 22 Jahren hat Pat, auf beiden Wangen und der Nase einen Ausschlag, der einem Lupus erythematosus ähnelt, gehabt. Vor ungefähr 9 Monaten bildete sich auf dem rechten Nasenflügel neben der Grenzfalte zur Wange ein kleines Geschwür, das seitdem nicht geheilt und nun kaum pfennigstückgroß ist, mit unbedeutend eingesenktem, krustenbelegtem Grunde und umgeben von einem niedrigen Wall von blasser Farbe und fester Konsistenz (Fig. I4).

8. V. Behandlung mit Kohlensäure unter kräftigem Druck während I Minute. I6. V. Erneute Behandlung, nach Ablösen der Kruste, während 2 Minuten. Nach der Behandlung trat eine Anschwellung der Umgebung, der Wange und der Augenlider auf der rechten Seite ein. 30. V. Noch immer findet sich ein ziemlich fester Wall um das in Heilung begriffene Geschwür herum. 6. VI. Das Geschwür ist nun vollständig geheilt mit glatter Epidermis, der umgebende Wall nahezu verschwunden. Die Narbe von derselben Farbe wie die Umgebung. Erneute Behandlung I Minute lang. 22. VI. Geheilt. (Siehe Fig. I5).

20. VII. Andauernd geheilt; über die im übrigen fast unsichtbare Narbe läuft ein schwach angedeuteter, millimeterbreiter, fester, narbenähnlicher Wulst; möglicherweise Rezidiv, weshalb der verdächtige Streifen mit der Schneide eines keilförmigen Kohlensäurestabes behandelt wurde.

22. IX. Ançauernd geheilt. Die Narbe so gut wie unsichtbar, glatt, im Niveau der umgebenden Haut und von derselben Farbe wie diese, sic h von ihr nur durch größere Glätte unterscheidend.

8. XI. Keine Veränderung.

Der Fall kildet ein schönes Beispiel für die guten Resultate der Gefri ermethcde kei dem gewöhnlichen, oberflächlichen Ulcus rodens. Heilurg trat binn€n eines Monats ein, während welcher Zeit die Patientin dreimal, bzw. 30, 60 und 30 Sekunden lang

I) S. Handley, Cancer of the breast and its operative treatment, Lond on 1906. 
behandelt worden war. Nach der Heilung des Geschwürs wurden noch einige Partien der Narbe behandelt, die möglicherweise

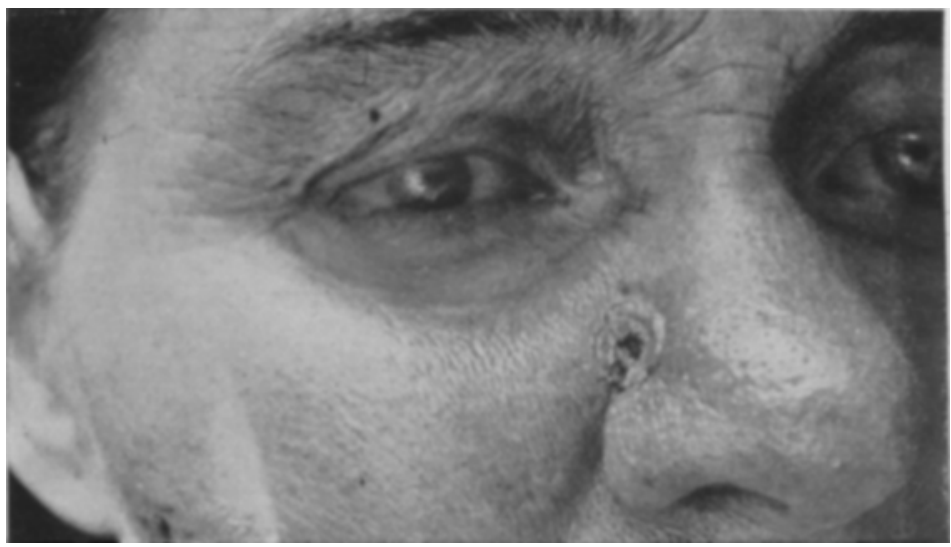

Fig. I4.

Krebselemente enthalten konnten. Die Heilung erweist sich nach $3 \frac{1}{2}$ Monaten als beständig, und das kosmetische Resultat ist sehr schön.

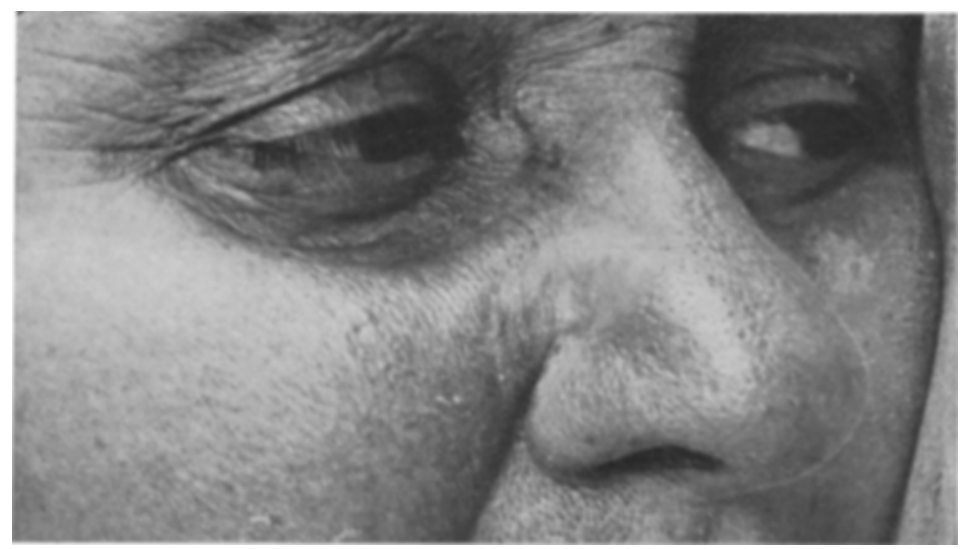

Fig. I 5 .

Nr. 7. Augusta H., 57 J., unverheiratet. Serafimer-Lazarett, chir. Abt. I, Nr. 48I, IgII.

Ulcus rodens nasi. Pathol.-anat. Diagnose: Krebs. 
Vor ungefähr 5 Jahren trat ein kleines Geschwür mitten auf dem Nasenrücken auf, das dann zeitweise geheilt gewesen ist, im letzten Halbjahre aber um sich zu greifen begonnen hat. Nun

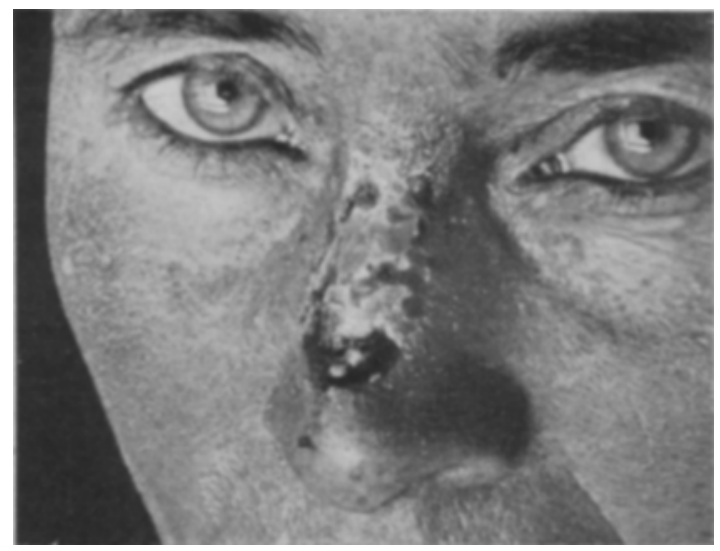

Fig. 16.

finden sich auf der Nase 3 Geschwüre, voneinander getrennt durch narbig umgewande te Haut; zwei von den Geschwüren haben

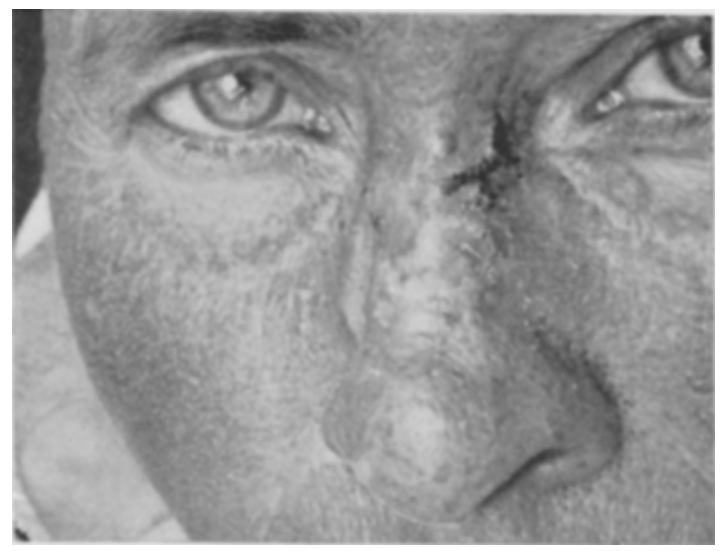

Fig. 17 .

girlandenförmige Konturen und zeigen das Bild serpiginöser Ulcerationen (Fig. I6).

Io. VI. Behandlung der größeren Geschwürsoberflächen während I Minute, der kleineren während $1 / 2$ Minute. 
2r. VI. Das oberste, kleinste Geschwür geheilt, die übrigen in guter Heilung begriffen. Erneute Behandlung der noch vorhandenen Geschwüre während $30-60$ Sekunden.

I7. VII. (Fig. I7.) Alle behandelten Geschwüre schön geheilt mit feiner, von der Umgebung wenig abweichender Narbe. An der Nasenwurzel aber auf jeder der beiden Seiten, wo Geschwüre vorher nicht beobachtet worden waren, eine kleine, unregelmäßige, bandförmige, krustenbelegte Ulceration, die $\mathrm{I}^{1 / 2}$. Minuten lang behandelt wurde.

26. VII. Ein ungefähr doppelt stecknadelkopfgroßer Knoten in der Haut, ohne Ulceration, belegen auf der rechten Seite der Nasenwurzel, nahe einer der obenerwähnten Ulcerationen wurde $I^{1 / 2}$ Minuten lang behandelt, ein etwas größerer, ähnlicher Knoten mitten auf dem Nasenrücken (innerhalb der Narbe einer spontan geheilten Ulceration ?) wurde einige Tage später behandelt. Schließlich wurden am 4. VIII. ein ganzer Kranz von kleinen, kaum millimeterdicken oder noch kleineren Wällen aus festem Gewebe an der Peripherie der geheilten Geschwürspartien, der sich bis über das Lig. palpebr. mediale beiderseits von der Nasenwurzel aus hinüber erstreckte, sowie ähnliche Partien weiter unten längs den Seiten der Nase 30-9o Sekunden lang behandelt.

Pat. wurde als geheilt am 27. VIII. entlassen und ist laut brieflicher Mitteilung noch am Ig. III. IgI2 völlig geheilt.

Eine Heilung der vorhandenen, sehr oberflächlichen Ulcerationen wurde in diesem Falle durch $\mathrm{I}$ oder 2 Gefrierungen von 30-60 Sekunden bewirkt. Einige kleinere, später auftretende Ulcerationen sowie zahlreiche kleine, wallartige Infiltrationen an den Rändern der geheilten Geschwürsflächen machten einige weitere Behandlungen erforderlich. Das Resultat war in kosmetischer Beziehung ausgezeichnet und noch nach 7 Monaten bestehend.

Nr. 8. Johann Alfred A., 66 J., Tischler. Serafimer-Lazarett, chir. Poliklinik, I9II, Nr. 3890.

Ulcus carcinomatosum reg. infraorbitalis sin. Pathol.anat. Diagnose: Plattenepithelkrebs.

Pat. hatte vor 2 Jahren, im Frühling und Sommer I9o9, ein kleines chronisches Geschwür auf der linken Wange mit typischem Charakter von Hautkrebs. Verf. behandelte dasselbe mit Wärme von so hohem Grade, wie der Patient sie ertragen konnte (mehrere Sitzungen von je I Stunde; die Wärme wurde dem Geschwür mittelst einer kleinen Bleiröhre zugeführt, durch die heißes Wasser zirkulierte). Das Geschwür heilte, trat aber im Frühling IgII wieder auf. Es bildet nun (April) eine erbsengroße Grube, umgeben von einem festen Wall (Fig. I8). 
Die Wärmebehandlung wurde aufs neue versucht, ergab aber kein Resultat. Nach Gefrieren mit Kohlensäure 2 Minuten lang, Ende Juni, heilte das Geschwür binnen ei niger Wochen. Die Narbe wurde

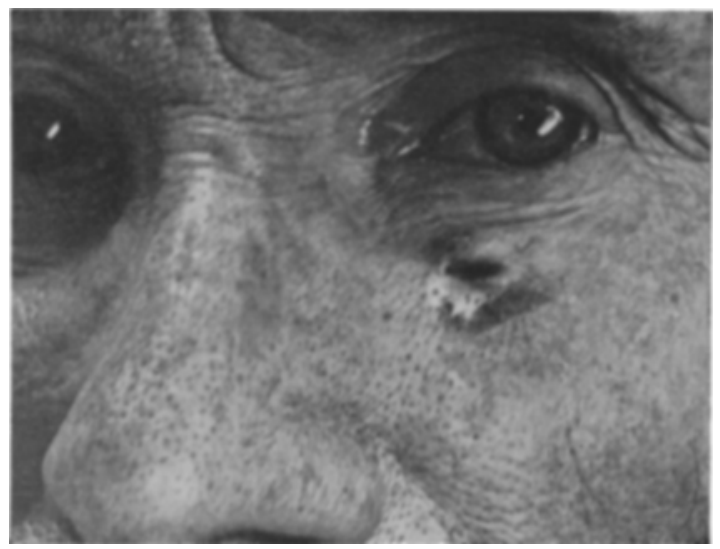

Fig. I8.

noch eine weitere Minute lang behandelt. Fig. I9 zeigt das geheilte Geschwür ungefähr $\mathrm{I}^{1 / 2}$ Monate nach der ersten Behandlung. In der

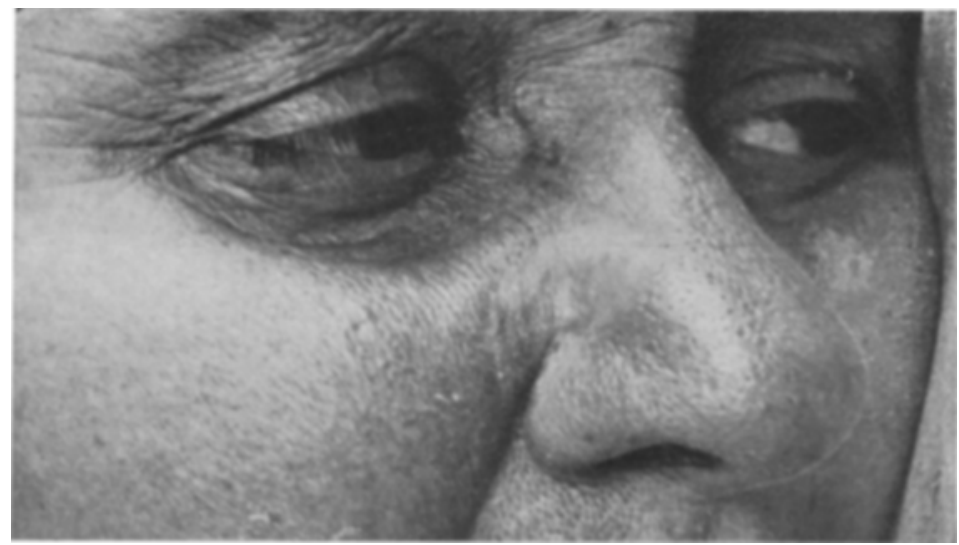

Fjg. 19.

Umgebung der Narbe fühlte sich die Haut damals noch etwas infiltriert an, bei Nachuntersuchung am 3I. X. aber, $3^{1 / 2}$ Monate nach der Heilung, war sie überall weich und fein. 
Nr. 9. Mathilde A., 46 J., unverheiratet. Serafimer-Lazarett, chir. Poliklinik, I9II, Nr. 6998.

Ulcus carcinomatosum faciei. Pathol.-anat. Diagnose: Plattenepithelkrebs.

Seit einigen Jahren ein kleines Geschwür auf der rechten Wange neben der Mittelpartie der Nase. Das Geschwür wurde vor I $1 / 4$ Jahren excidiert, rezidivierte aber. Nach Mitteilung der Pat. ist sie in einem Provinzkrankenhaus erfolglos mit Röntgen behandelt worden. Sie hat nun ein kaum erbsengroßes, leicht blutendes Geschwür an der angegebenen Stelle, nach unten zu und auf beiden Seiten umgeben von einem schwach angedeuteten, grauweißen Walle von fester Konsistenz. Das Geschwür liegt innerhalb einer narbig umgewandelten Partie der Haut (von der Excision her).

28. VI. Behandlung 3 Minuten lang. Am folgenden Tage Anschwellung der behandelten Partie und ihrer Umgebung, das rechte Auge nicht zugeschwollen; die gefrorene Partie blaurot mißfarbig mit einigen kleinen hämorrhagischen Bläschen am Rande. Erneute Behandlung I Minute lang, worauf Pat. aufs Land zurückreisen durfte.

I8. VII. Geheilt mir feiner, weicher Narbe von der Größe einer Erbse, etwas röter als die Umgebung, die in einem millimeterbreiten Ringe nächst dem Geschwür vielleicht etwas fester ist als die Haut auf der Wange im übrigen. Erneute Behandlung I Minute lang.

27. X. IgII und 20. III. IgI2. Laut brieflicher Mitteilung andauernd geheilt und im übrigen keine Veränderung.

Dieser Fall ist insofern von Interesse, als ein Rezidiv nach der Excision eingetreten und nach Angabe der Pat. Röntgenbehandlung versucht worden war, ohne jedoch ein Resultat zu ergeben, was indessen viclleicht auf mangelhafter Technik beruht haben kann. Das Geschwür heilte vollständig binnen 20 Tagen, nachdem Pat. eine sehr kräftige Behandlung mit Kohlensäure, nämlich 3 Minuten lang in eincr Sitzung und weitere I Minute bereits am folgenden Tage erhalten hatte. Dic Heilung noch nach 8 Monaten von Bestand.

Nr. 10. Johanna J,, 49 J., Ehefrau. Serafimer-Lazarett, chir. Klinik I, I9II, Nr. 63I.

Cancercutis reg. cubitisin. Pathol.-anat. Diagnose: Plattenepithelkrebs.

In der Narbe nach einer schwereren Brandverletzung, die sich Pat. im Alter von 4 Jahren zuzog, entstand vor 4 Jahren ein Geschwür, das dann zeitweise geheilt gewesen, nun aber seit einem $3 / 4$ Jahr offensteht und an Größe zugenommen hat. Jetzt ein kinderhandgroßes, unregelmäßiges Geschwïr, mit eitrigen Granulationen, leicht blutender 
Oberfläche und mäßig erhabenen, nekrotischen Rändern. Starke Narbenkontraktur im Ellenbogengelenk.

2I. VIII. Nach Wegkratzen aller lockeren Massen hinab bis auf festes Gewebe einige Millimeter unter dem Niveau der Hautoberfläche wurden ungefähr $\% \frac{1}{3}$ des Geschwürs einschließlich der Ränder mit großen, zweckmäßig zugeschnittenen Stücken von Kohlensäure 5 Minuten lang behandelt.

Im Laufe der nächsten 2 Monate trat Epidermisierung am Rande eines Teils des behandelten Gebietes ein; im übrigen keine Heilung. Neuralgien im Arm. Amputatio humeri 14. X.

Nr. 11. Gustav J., 54 J., Kupferschmied. Serafimer-Lazarett, chir. Poliklinik, IgII, Nr. Io 267.

Cancer cutis faciei. Pathol-anat. Diagnose: B asalzellenkrebs.

Ohne bekannten Anlaß entstand vor ca. 9 Jahren ein kleines Geschwür auf der rechten Wange, nachdem kurze Zeit vorher an derselben Stelle ein ,,roter Streifen" bemerkt worden war. Während der folgenden 3 Jahre unbedeutendes Wachstum des Geschwürs, das nicht größer als eine Erbse war. Nach Akbratzen und Finsenbehandlung im Krankenhaus zu St. Göran vor 6 Jahren, heilte das Geschwür und blieb $2 \frac{1}{2}$ Jahre lang geheilt, wonach es rezividierte, und seitdem hat es langsam an Größe zugenommen.

Jetzt findet sich auf der rechten Wange eine gut pfennigstückgroße Ulceration, belegt mit einer Kruste, bei deren Ablösung das Geschwür blutet. Der Geschwürsgrund im allgemeinen im Niveau der umgebenden Haut oder unbeträchtlich eingesenkt, aus granulationsähnlichem Gewebe von hellroter Farbe bestehend. Die Geschwürsränder nicht wallartig aufgetrieben.

II. IX. Behandlung während 2 Minuten. Danach bedeutende Anschwellung der rechten Wange, sich über den Nasenrücken hin auf die andere Seite erstreckend; das rechte Auge schwoll zu. Die Schwellung in den Augenlidern verschwand nach einigen Tagen. Die auf dem Geschwür gebildete Kruste fiel am 20. IX. ab, und das Geschwür zeigte sich nun, 9 Tage nach der Behandlung, überall außer auf einer kleinen Partie in der Mitte mit Epidermis bedeckt. Ein großer Teil des Geschwürsrandes zeigt eine weiße, wallartige Auftreibung. Erneute Behandlung während 2 Minuten.

30. X. Vollständig geheilt mit weicher Haut von derselben Farbe wie die Umgebung, mit einer kaum merkbaren Einsenkung unter die umgebende Hautoberfläche. Am Rande der Narbe einige kleine, höchstens stecknadelkopfgroße Unebenheiten in der Epidermis, weshalb die ganze Partie aufs neue 60 Sekunden lang behandelt wurde.

I0. II. I9I2 völlig geheilt; keine Zeichen eines Rezidivs.

Auch in diesem Falle, einem Basalzellenkrebs, wurde eine 
rasche Heilung des Krebsgeschwürs nach energischem Gefrieren erhalten. Die, erste Behandlung, 2 Minuten lang, hatte eine ungewöhnlich kräftige entzündliche Reaktion zur Folge. Die Behandlung wurde bereits nach 9 Tagen wiederholt, wo die Reaktion vollständig abgelaufen und die Kruste abgefallen war. Gute Heilung nach 7 Wochen konstatiert; einige noch zurückgebliebene kleine Unebenheiten in der Epidermis, wahrscheinlich ohne Bedeutung, wurden dennoch der Sicherheit wegen nochmals mit Gefrieren behandelt. Nach 5 Monaten keine Zeichen eines Rezidivs.

Nr. 12. Johannes L., 75 J., Arbeiter. Serafimer-Lazarett, chir. Poliklinik, IgI I, Nr. I2 522.

Cancer cutis reg. zygom. sin. Pathol,-anat. Diagnose: Krebs.

Seit I Jahre ein Geschwür auf der linken Jochbeingegend ungefähr $2 \mathrm{~cm}$ unterhalb und etwas nach außen vom äußeren Augenwinkel. Jetzt pfennigstückgroßes Geschwür mit etwas erhabenem Grunde, umgeben von einem niedrigen Wall von sehr festem Gewebe.

2. XI. Behandlung während 2 Minuten. Schon nach I Minute fühlte sich die dünne Haut an dem Knochen fest gefroren an. Pat. zeigte keine Zeichen von Schmerz, gab auf Befragen an, daß es nicht wehtäte.

II. XII. Das Geschwür geheilt mit unsichtbarer Narbe, außer einer kaum erbsengroßen Partie im Zentrum, die noch mit einer festsitzenden Kruste bdeckt ist, bei deren Ablösung eine kleine, unebene, oberflächliche Ulceration entblößt wird. Diese wurde noch weitere 2 Minuten lang behandelt. Die wallförmige Auftreibung an der Peripherie des Geschwürs ist vollständig verschwunden.

26. XII. Vollständig geheilt.

(Nachtrag zur Korrektur.) Juni IgI2. Nach mündlichen Mitteilungen andauernd geheilt.

Nr. 13. Frau L. R., 55 J. Serafimer-Lazarett, chir. Poliklinik I9II.

Cancer cut is nasi. Path.-anat. Diagnose: U1cus rodens.

Seit Io Jahren eine jetzt kaum pfennigstückgroße Erhöhung an der Nasenwurzel, von heller Farbe und sehr fester Konsistenz; hat niemals ulceriert. Erste Behandlung am 23. XI. IgII.

Geheilt mit kaum sichtbarer Narbe von derselben Farbe und dem gleichen Niveau wie die umgebende Haut nach 3 Behandlungen von $2-3$ Min. während $2^{1 / 2}$ Mon.

Nr. 14. Emma A., 6o J., unverheiratet. Serafimer-Lazarett, chir. Poliklinik I9I2, Nr. 204.

Cancer cutis nasi. Path.-anat. Diagnose: Bas a 1 zellcancer.

Seit mehreren Jahren eine während des letzten $3 / 4$ Jahres ulcerierende „Warze" an der linken Seite der Nase. Jetzt ein pfennigstückgroßes, 
etwas erhabenes Geschwür mit wallartig aufgetriebenem Rande, von dem typischen Aussehen des Ulcus rodens.

Behandlung am 9. I. und I6. I. I9I2, je 3 Min.

(Die Behandlung mußte auf eine Woche konzentriert werden, da die Pat. nur diese Zeit in Stockholm verweilen konnte.) Die Heilung wurde von Herrn Provinzialarzt Dr. Wa rodell in Östhammr gütigst überwacht. Er schreibt mir, daß das Geschwür am 9. II., also 24 Tage nach der letzten Behandlung, völ lig geheilt war." Die Haut darüber ist glatt und eben, ohne Retraktionen und ohne bestimmte Markierung gegen die Umgebung, nur durch eine schwache Rosafarbe von derselben abweichend. Eine ideale Heilung und ein ideales Resultat kann ich konstatieren."

Nr. 15. C. G. G., 7I J., ehem. Kaufmann. Serafimer-Lazarett, chir. Poliklinik Igr2, Nr. I983.

Cancer cutis faciei. Path.-anat. Diagnose: Basalzellcarcinom.

Seit etwa 8 Jahren Geschwüre an der linken Wange, die im Krankenhause zu St. Göran während der ersten 4 Jahre mittelst Auskratzung und Finsenbehandlung (I904, I905, I907 und I908 je 4, 3, 8 und Io Sitzungen), dann mittelst Röntgenbehandlung (IgIo und I9II insgesamt I6 Sitzungen) mehrmals vorübergehend geheilt worden ist. Vor 7 Mon. auch zweimalige Radiumbehandlung.

Jetzt 2 Gruppen von krustenbedeckten Geschwüren an der linken Wange; die eine, an der Nasenseite, besteht aus einem bohnengroßen und zwei stecknadelkopfgroßen Geschwüren, die andere, auf der Mitte der Wange, besteht aus 4, höchstens hanfkorngroßen Geschwüren. Die Ulcerationen sind etwas eingesenkt, mit scharfen, nicht besonders harten Rändern und unebenem, eitrig belegten Grunde, der sich ziemlich wenig infiltriert anfühlt.

23. II. Die Geschwürsgruppe an der Nase wurde auf einmal 3 Min. gefroren, die andere Gruppe auf einmal 2 Min. Geheilt nach 3 Wochen.

(Nachtrag zur Korrektur.) 4. VII. I9I2. Geschwüre andauernd geheilt, ohne Zichen von Rezidiv in den Narben. Patient ist sehr zufrieden, da nach keiner der früheren Behandlungsmethoden die Heilung so lange ge lauert hat. Ein neues hanfsamengroßss Geschwür neben dem linken Nasenflügel wird 3 Min. lang behandelt.

Nr. 16. G. I.

Ulcus rodens faciei.

Seit etwa I Jahre ein gut erbsengroßes Geschwür an der linken Wange, von dem für das Ulcus rodens typischen Aussehen.

I9. III. Behandlung 2 Min.

9. IV. Geheilt mit etwas eingesenkter Narbe. Zwei kleine Epidermisverdickungen am Rande der Narbe wurden am 22. IV. 30 Sek. efroren. 
26. VI. Die Narbe schwach erhaben, von derselben Farbe wie die Umgebung. Am Rande noch eine stecknadelkopfgroße Epidermisverdickung.

Im Zusammenhang mit den eben angeführten Fällen von Hautkrebs dürfte es angebracht sein, einen Fall von $\mathrm{H}$ a u $\mathrm{t}$ e nd oth eliom zu erwähnen, welche Geschwulst ja klinisch dem Hautkrebs nahesteht.

Nr. 17. Edla E., 6o J., Ehefrau. Serafimer-Lazarett, chir. Poliklinik, I9II, Nr. 9567 .

A) Tumor exulcerans reg. nuchae. Path.-anat. Diagnose: Endotheliom.

B) Ulcus chron. nasi. Path.-anat. Diagnose: En dotheliom.

A) Seit 25 Jahren eine kleine Warze dicht unterhalb und hinter dem Proc. mastoideus dexter. Die Warze ist oft beim Kämmen abgerissen worden und hat dabei geblutet. Seit 5-ro Jahren hat sie an Größe zugenommen. Sie hat nie geschmerzt außer in der letzten Zeit, wo bisweilen Stiche in derselben verspürt wurden.

An der angegebenen Stelle erhebt sich ungefähr $1 / 2 \mathrm{~cm}$ über die umgebende Haut eine etwa zweipfennigstückgroße, kleinknotige Geschwulst von schmutzigroter Farbe, ulceriert und hier und da mit kleinen, graugelben Krusten bedeckt. Die Geschwulst fühlt sich ziemlich fest an und zeigt im Durchschnitt eine graurote Masse ohne festeres verbindendes Stroma (Fig. 20). Behandlung am 25. VIII. Die Geschwulst wurde mit scharfem Löffel bis auf das Niveau der Haut weggekratzt. Der Geschwürsgrund wurde mit Kohlensäure 3 Minuten lang behandelt, was starken Schmerz hervorrief. II. IX. Das Geschwür rein und schön mit niedrigen Granulationen von gesundem Aussehen, zusammengezogen, mit guter Heilung von den Rändern her. Erneute Behandlung einer etwas erhabenen Partie im Geschwürsgrunde während 2 Minuten. I7. X. Geheilt. Neben dem Rande der Narbe eine hanfkorngroßer blasser Knoten, einem kleinen verrukösen Naevus ähnelnd (Fig. 2I). Behandelt $30+90+30$ Sekunden mit Auftauen zwischen jeder Applikation.

B) Eine kleine „Warze“ auf der rechten Seite der Nasenwurzel seit ungefähr 5 Jahren; seit einigen Monaten an derselben Stelle ein Geschwür, das allmählich an Größe zugenommen hat, ohne Schmerzen zu verursachen. Jetzt eine Ulceration von dem für Ulcus rodens carcin. typischen Aussehen, kaum pfennigstückgroß, mit unebenem, krustenbelegtem Grunde, umgeben von einem hellgrau gelben, schwach erhabenen Wall, der bis über das Lig. palpebrale mediale hinausreicht. (Fig. 22).

25. VIII. Behandlung mit Kohlensäure 2 Minuten lang. Starker Schmerz. II. IX. Geheilt mit ebener und schöner, noch etwas geröteter Narbe. I7. X. Andauernd geheilt (Fig. 23), aber eine kleine verdächtige 
Erhebung am Rande der Narbe, die $30+$ I5 + I5 Sekunden lang mit Auftauen zwischen jeder Applikation behandelt wurde.

An beiden Stellen hat sich das Endotheliom wahrscheinlich wie gewöhnlich auf der Basis eines Naevus verrucosus entwickelt. Bei der Behandlung der ziemlich dicken Geschwulst im Nacken

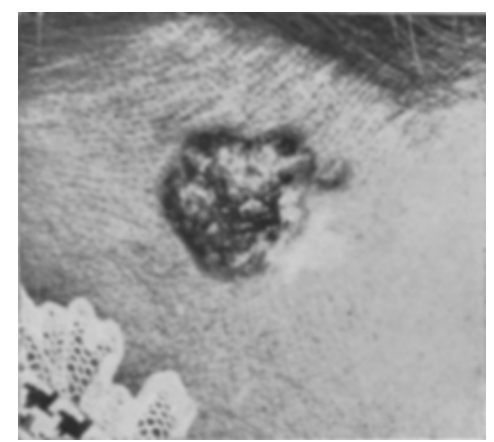

Fig. 20.

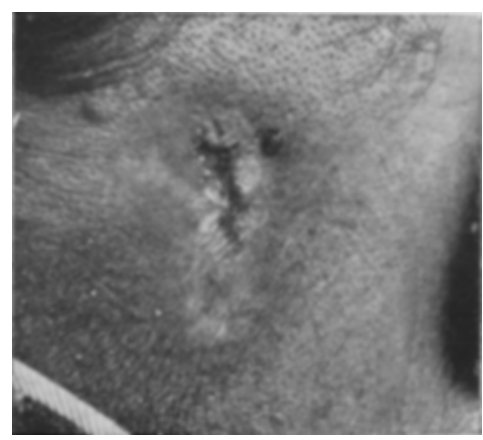

Fig. $2 \mathrm{I}$.

erachtete ich für das beste, zuerst eine gründliche Auskratzung vorzunehmen, um leichter die tiefsten Teile der Geschwulst durchfrieren zu können. Es ist ja möglich, daß das Auskratzen allein zur Herbeiführung der Heilung hinreichend gewesen wäre, und

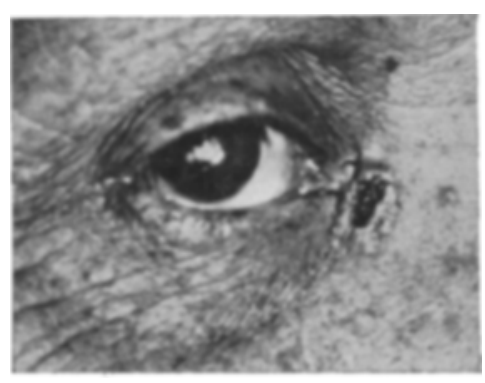

Fig. 22.

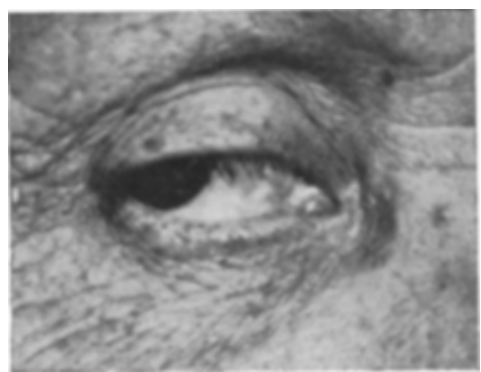

Fig. 23.

der Fall kann demnach kaum als Beweis für die Effektivität des Gefrierens angeführt werden.

Bei dem Endotheliom B wurde nur Gefrieren in einer einzigen Sitzung von 2 Minuten ausgeführt, das Heilung binnen I7 Tagen bewirkte; eine kleine übriggebliebene Verdickung am Narbenrande wurde erneutem Gefrieren nach 2 Monaten unterzogen. 
Bemerkenswert ist in diesem Falle der starke Schmerz, den das Gefrieren hervorrief; der Schmerz verschwand zwar bereits einige Minuten nach der Behandlung, war aber doch so stark, daß die Patientin sich eine Form der Betäubung ausbedang, wenn die Behandlung wiederholt werden sollte.

Der Vollständigkeit wegen wäre vielleicht in diesem Zusammenhang ein mit Kohlensäure-Gefireren behandelter Fall von $\mathrm{H}$ a u $\mathrm{tkrebs}$ m it $\mathrm{H}$ a u t metastasen zu erwähnen. Der Fall ist von sehr großem Interesse, doch vielleicht mehr als Beispiel für die relative Gutartigkeit des Krebses bei hohem Alter, denn als weiterer Beitrag zu dem, was die Gefrierbehandlung bezüglich der Heilung desselben $\mathrm{zu}$ erreichen vermag.

Nr. 18. Maria O., 84 J., unverheiratet. Offentl. Versorgungsanstalt, Westpavillon, IgIr, Nr. II7.

Cancer buccae sin. cum metastas. subcutan. faciei et reg. inguin. sin. - Path.-anat. Diagnose (Muttergeschwulst und Leistenmetastase): Krebs.

Pat. wurde im Krankenhaus Sabbatsberg im Sommer rgro wegen Hautkrebs auf der linken Wange behandelt. Ein kleineres Ulcus carcinomatosum war vorher in der Poliklinik exstirpiert worden, und nun fand sich an derselben Stelle eine haselnußgroße, gegen die Unterlage verschiebbare Geschwulst nebst einigen kaum erbsengroßen Metastasen in der Haut, welche sämtlichen Geschwïlsten exstirpiert wurden. Ein Rezidiv trat in der Narbe auf, und Pat. wurde, nachdem sie einige Monate hindurch erfolglos mit Röntgen behandelt worden war, als inoperabel am 2I. I. IgII in das Krankenhaus der Offentl. Versorgungsanstalt aufgenommen. Fig. 24 gibt eine Vorstellung von dem Zustande im März IgIı. Außer der großen ulcerierenden Geschwulst in der linken Wange hat sie 5 subkutane Hautmetastasen (Fig. 24, I, 2, 3, 4, 5 , einige ulcerierend, die übrigen noch mit Haut bedeckt. Probeweise waren in der Zeit vom I3. II. - I8. III. 3-4 Gefrierungen von 30-60 Sekunden an den zwei kleinen, dem Ohre nächstliegenden Metastasen ausgeführt worden (Fig. 24, I und 2; die Krusten nach der Behandlung noch vorhanden). Sie verschwanden nach einigen Wochen, nur einen kleinen Pigmentfleck zurücklassend, der später auch allmählich verschwand. Nun wurden auch die übrigen behandelt. Auf jeder der beiden größeren Metastasen wurde einige dicke Kohlensäurestäbe 5 Minuten lang appliziert. Durch eine partielle Excision des einen Knotens unmittelbar nach der Behandlung wurde konstatiert, daB das Gefrieren ungefähr $6 \mathrm{~mm}$ in die Tiefe gedrungen war. Die gefrorenen Partien fielen $a b$, und die Behandlung wurde an den zurückbleibenden, 
noch etwas über die Umgebung sich erhebenden Geschwürsoberflächen wiederholt. Es trat langsam vollständige Heilung mit so schöner Narbenbildung ein, daß sie kaum von der umgebenden Haut unterschieden werden kann. Fig. 25 ist am I. VIII. aufgenommen, wo die letzten zwei Geschwüre nach den Metastasen in Heilung begriffen sind, während die Hauptgeschwulst andauernd an Größe zugenommen hat und über die ganze Oberfläche hin in Ulceration begriffen ist.

Unter Lokalbetäubung exstirpierte ich nun am I8. VIII. die große Geschwulst mit Thermokauter. Gleichzeitig wurde in dem Anschein

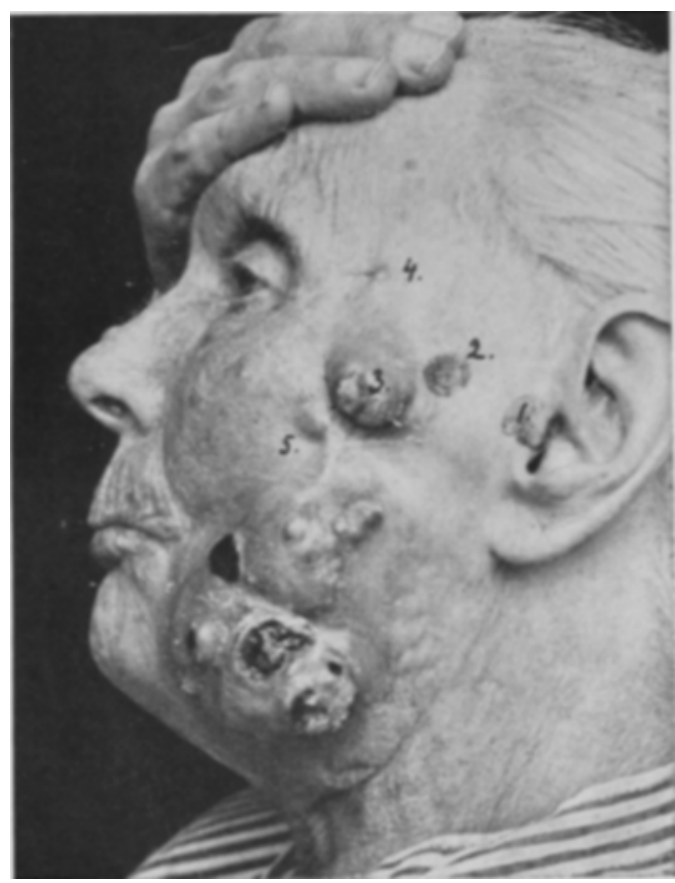

Fig. 24 .

nach gesundem Gewebe eine pflaumengroße Metastase in der Haut oberhalb der linken Leiste exstirpiert. Das Geschwür zog sich rasch zusammen, in seinem Grunde aber sproßten in kurzer Zeit drei wohlbegrenzte bohnengroße Knoten empor, offenbar Rezidive (Fig. 26; man sieht dort die Geschwüre, die nach den mit Kohlensäure behandelten Metastasen entstanden waren, völlig geheilt). Die erwähnten Knoten wurden mit scharfem Löffel weggekratzt und die Geschwürsgründe anch dem Auskratzen mit Kohlensäure behandelt. Die Heilung des Geschwürs machte nun rasche Fortschritte, sie war am 30. IX. voll- 
ständig und erwies sich nach einem Monat (28. X.) als von Bestand (Fig. 27); nach einer weiteren Zeit von mehr als einem Monat kein Zeichen von Rezidiv. In der im übrigen ebenen und schönen Narbe findet sich indessen eine Parotisgangfistel als Merkmal nach der tiefen Zerstörung in der Wange.

(Nachtrag zur Korrektur.) 3. VII. I9I2. Narben weich, sehr schön; nirgends Zeichen von Rezidiv. Die Parotisgangfistel geheilt.

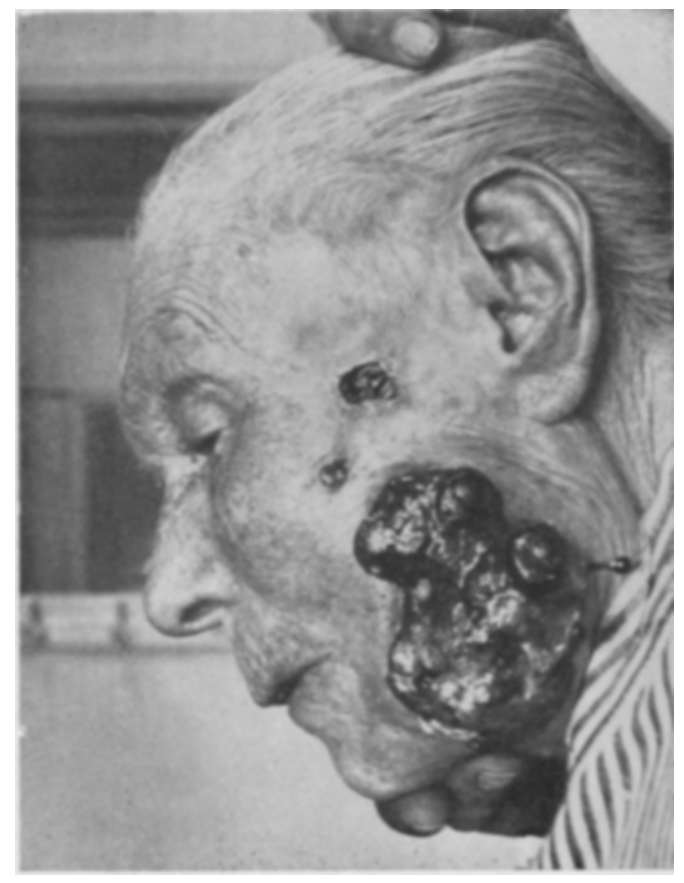

Fig. 25 .

In sämtlichen Fällen mit Ausnahme von $\mathrm{Nr}$. I6 ist die Diagnose Hautkrebs mikroskopisch festgestellt worden; das Aussehen des Geschwürs in Nr. I6 war ganz typisch, so daß auch hier die Diagnose kaum in Zweifel gezogen werden kann.

Betreffs der Behandlung glaube ich, daß ein energisches Verfahren schon bei der ersten Applikation vorzuziehen ist. Die Tiefe der Cancervegetationen ist nicht leicht $\mathrm{zu}$ bestimmen, und man tut also gut, von Beginn an das Gefrieren in die größtmögliche Tiefe, die durch die von der Neubildung eingenommene 
Fläche hindurch ohne allzu starke Reizerscheinungen erreicht werden kann, zu treiben.

$\mathrm{Zu}$ diesem $Z$ wecke wird am besten die ganze Geschwürs$\mathrm{f} l$ ä $\mathrm{ch}$ e a u f e in ma 1 unter starker Kompression behandelt. Gewöhnlich brauche ich eine Applikationszeit von 2-3 Minuten und wiederhole die Behandlung nötigenfalls nach 2-3 Wochen. Bei sehr oberflächlichen Geschwüren kann eine einzige Behandlung während 30 Sekunden genügen, doch scheint es mir klug, niemals unter eine Minute hinabzugehen, da eine mäßige Überdosierung nicht schadet.

Nach Gefrieren während 3 Minuten an der Wange tritt gewöhnlich eine Schwellung der umgebenden Haut ein, und das Auge kann für einen oder ein paar Tage zuschwellen, worauf der Pat. vorbereitet werden sollte.

Wenn ich nun zum Schluß die Resultate der Behandlung zusammenstelle, so könnte der letzterwähnte Fall ja insofern der Kategorie Hautkrebs, behandelt mit Kohlensäure, zugerechnet werden, als ja auch Rezidive der Hauptgeschwulst Gegenstand der Gefrierbehandlung gewesen sind,

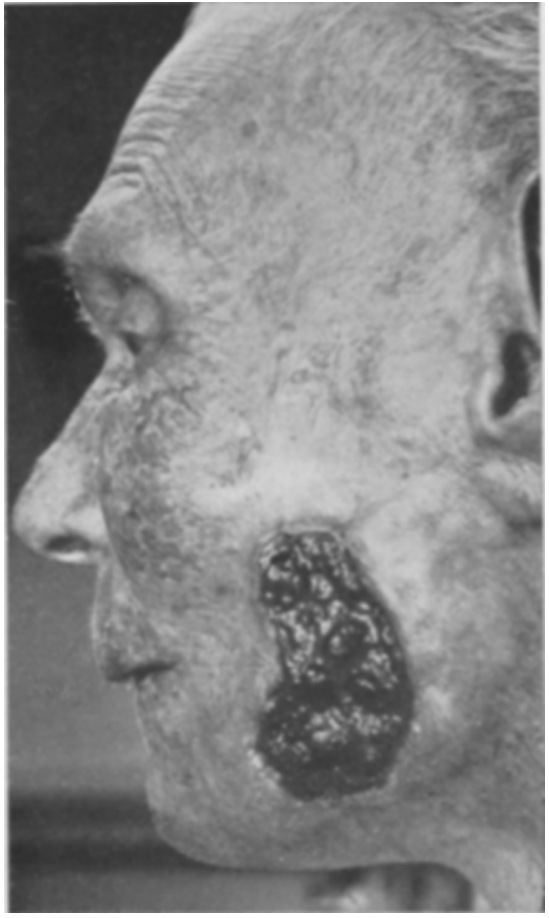

Fig. 26. der Fall nimmt aber in mehreren Hinsichten eine Sonderstellung ein ${ }^{1}$, und es erschien mir daher am zweckmäßigsten, ihn nicht mit den übrigen in eine Reihe, zu stellen.

I) Unter anderem hatte Pat. während 4 Monaten, vom I. II. an, Injektionen von Autolyseprodukten von menschlichem Embryonach Fichera erhalten. Fichera will, wenn ich ihn richtig verstanden habe, 
Wenn wir dagegen das Endotheliom als eine Art Hautkrebs rechnen, so ist bei einer Zusammenstellung der Behandlungsresultate auch der Fall Nr. I7 mitzuzählen und zweckmäßigerweise wegen der verschiedenen klinischen Beschaffenheit der beiden Geschwülste in 2 Fälle zu teilen. Wir erhalten dann I8 Fälle von Hautkrebs. In Nr. I und Io wurde die Behandlung nur begonnen. Aus dem Verlauf bei den übrigen I6 können aber Schlüsse über den Wert der Kohlen-

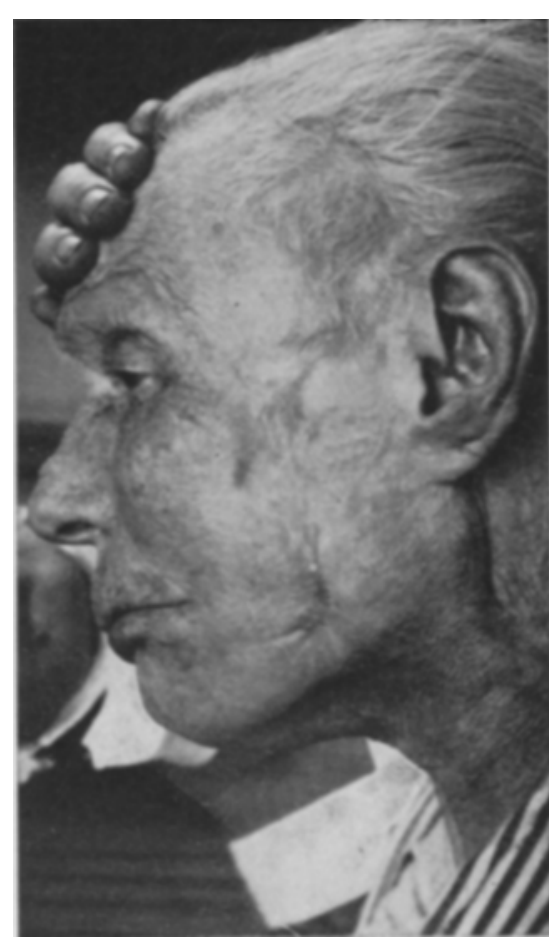

Fig. 27. säurebehandlung gezogen werden. Das Material ist ja nicht sehr groß, die Resultate aber scheinen mir in eine bestimmte Richtung zu weisen - bis auf weiteres.

Von den I6 Fällen sind I3 oberflächliche Formen, die meisten von dem gewöhnlichen Typus für Ulcus rodens; diese sind sämtlich im Laute einiger Wochen loder höchstens einiger Monate in vereinzelten Fällen vollständig geheilt. Zwei von den Fällen sind mehr bösartige, tiefgehender Fomen, von überwiegend ulcerativer Natur; in beiden ist Heilung eingetreten, aber nur unter sehr langwieriger und energischer Behandlung; in dem einen Falle, wo die Behandlung abgebrochen worden ist,

in dem Autolysat von Epithelteilen von Embryos ein Ferment erhalten haben, daß das Vermögen besitzt, embryonale und epitheliale Elemente aufzulösen, und er hat nach längerer Zeit fortgesetzten Injektionen von Autolysat bei inoperablem Krebs Degenerationserscheinungen (besonders histologische) in der Krebsgeschwulst gesehen, die er mit den Injektionen in Zusammenhang stellt. Wir haben im Krankenhause der Öffentl. Versorgungsanstalt diese Methode in mehreren Fällen geprüft, bisher aber kein Resultat beobachtet - außer m ö g li c h e r w e is e in dem oben erwähnten Falle. 
wurde die Heilung nicht vollständig, in dem anderen ist die Heilung durch Auskratzen oder partielle Excision unterstützt und allmählich vollständig geworden. In diesen 2 Fällen ist demnach das Resultat zwar so befriedigend gewesen, da $\mathrm{B}$ es mir berechtigt erschien, die einmal begonnene Behandlung fortzusetzen, diese aber hat sich keineswegs als so kräftig oder zuverlässig erwiesen, daß sie in derartigen Fällen gewöhnliche operative Behandlung ersetzen oder den Wettbewerb mit den radiologischen Methoden aufnehmen zu können scheint - wo solche zugänglich sind. Schließlich haben wir das geschwulstförmige Endotheliom in Fall Nr. I7, das nach kurzer Zeit heilte, wo aber der Anteil des Gefrierens an dem Resultat wegen der ausgeführten Auskratzung unsicher ist.

Als ein bedeutsamer Gewinn bleibt die rasche Heilung in $\mathrm{s}$ ä $\mathrm{m} t \mathrm{lich}$ e $\mathrm{n}$ Fällen von oberflächlichem Hautkrebs bestehen. Daß eine Heilung in derartigen Fällen mit nahezu jedem beliebigen Kaustikum erreicht werden kann, dafür gibt es zahlreiche Beweise; ältere Versuche mit derartigen Mitteln scheinen sich aber nie ein allgemeineres Vertrauen erworben zu haben, sei es nun daß sie als allzu schwach und langsam wirkend befunden worden oder schmerzhaft gewesen sind oder häßliche Narben zurückgelassen haben. In allen diesen Hinsichten hat es sich gezeigt, daß die Gefriermethode hochgestellten Ansprüchen genügt. Die geradezu idealen kosmetischen Resultate dürften dem Umstande zuzuschreiben sein, daß das Gefrieren bei geeigneter Dosierung die epithelialen Elemente vernichtet, nicht dagegen das Bindegewebsstroma zu zerstören vermag, wodurch seine Wirkung in gewissem Grade e $1 \mathrm{e} \mathrm{kt} \mathrm{iv} \mathrm{ist,} \mathrm{was} \mathrm{ja} \mathrm{das} \mathrm{erstrebte}$ Ideal einer Behandlungsmethode gegen Krebs ist.

Nun muß indessen betont werden, daß es noch $\mathrm{zu}$ früh ist, sich über den dauernden Wert der Resultate zu äußern. Der älteste meiner Fälle ist nur I4 Monate lang nach der Heilung verfolgt worden. $\mathrm{Daß}$ auch eine dem Äußern nach vollständige Heilung von Hautkrebs sich als unvollständig durch ein spät auftretendes Rezidiv erweisen kann, ist wohlbekannt, und mein Fall Nr. 9 liefert ein Beispiel dafür; in den Fällen aber, die ich während längerer Zeit nach der Kohlensäurebehandlung habe verfolgen können, gewähren die ungewöhnlich schönen, fast unsichtbaren Narben, ohne Spuren einer Infiltration in der Haut 
oder andere Veränderungen in der Richtung auf ein Rezidiv, die beste Hoffnung, daß die Resultate von Bestand sein werden. Solchenfalls werden wir in dem Kohlensäuregefrieren eine neue Behandlungsmethode gegen Hautkrebs gewonnen haben, die wegen I. ihrer Ungefährlichkeit, 2. Einfachheit der Anwendung, 3. der Möglichkeit, exakt zu dosieren, 4. relativer Schmerzlosigkeit, 5. rascher Wirkung, 6. des kosmetischen idealen Resultats und 7. ihrer Billigkeit gecignet ist, die Normalmethode bei den oberflächlichen Formen der Krankheit zu werden, was von um so größerer Bedcutung wäre, als diese die überwiegende Mehrzahl der Fälle von Hautkrebs ausmachen und möglicherweise auch die Anzahl der jeder Art von Behandlung viel unzugänglicheren tiefgehenden Formen reduziert werden könnte, wenn jeder Arzt mit größter Leichtigkeit den Hautkrebs in seinem Beginn heilen könnte.

Stockholm, März I9I2.

\section{Literaturverzeichnis.}

I. Burns, Generalized multiple pigmented naevi, showing the result of two years treatment with liquid air and carbon dioxide snow. Journ. cut. diseases I9 Io, Bd. 28 , S. 690.

2. D a d e, Fall von Lupus erythematosus usw., behandelt mit Kohlensäure. Diskussion nach einem Vortrage von $\mathrm{H} \mathrm{u} \mathrm{b} \mathrm{b} \mathrm{a} \mathrm{r} \mathrm{d.} \mathrm{Journ.} \mathrm{of} \mathrm{cutan.} \mathrm{diseases}$ I 908 , S. I 37 .

3. Fr ü nd, H., Zur Technik der Kohlensäureschneebehandlung. Münch. med. Wochenschr. I9II, Bd. I.

4. Gottheil, Rodent ulcer of the lip; results of carbon dioxide treatment. Journ. cut. diseases I 909, 3d. 27, S. I 27.

5. Gottheil, William, Solid carbon dioxide in lupus erythematosus; a new and succesful method of treatment. New York med. Journal 1 go9, July 3.

6. Hochh a u s, H., Über Gewebsveränderungen nach lokaler Kälteeinwirkung. Virchows Archiv, Bd. I54.

7. Hoffm a n n, E. und $\mathrm{H}$ a $11 \mathrm{e}$, A., Demonstration der Behandlung eine; Naevus vasculosus mit Kohlensäureschnee-Erfrierung. Verh. der deutschen dermatolog. Gesellschaft, X. Kongr.

8. $\mathrm{H} \mathrm{u} \mathrm{b} \mathrm{b} \mathrm{a} \mathrm{d}, \mathrm{D}$., Method of making snow from liquid carbon dioxide for dermatological and surgical use. Journ. of cutan. diseases I908, S. 134 .

9. Derselbe, An apparatus for the rapid and economic making of $\mathrm{CO}_{2}$ snow. Journ. cutan. diseases I go8, S. 239.

xo. J a ckson, G. and $\mathrm{Hub}$ ba $\mathrm{rd}$, D., Freezing as a therapeutic measure; liquid air and carbonic acid snow. Med. Record. Apr. I909. Bd. 75, Nr. 16, 
I I. J u I i u s berg, M., Gefrierbehandlung bei Hautkrankheiten. Berl. klin. Wochenschr. I905, Nr. Io.

I2. Kuznitsky, Martin, Tubus zur Behandlung mit Kohlensäureschnee. Münch. med. Wochenschr. I9i I, Nr. io.

I3. Macleod, J. M. H., On the therapeutic value of carbon dioxide snow in the treatment of vascular naevi, moles etc. Brit. med. Journ. I9Io, Jan. 29.

I4. Morton, E. R., The treatment of naevi and other cutaneous lesions by electrolysis, cautery and refrigeration. Lancet I909, Dec. 4.

I5. D e r s e l b e , Some results obtained from the local application of solid carbon dioxide. Brit. med. Journ. I9Io, Jan. 29.

16. Nobl und Springels, Über die dermatotherapeutischen Anzeigen der Kohlensäureschneebehandlung. Zeitschr. für physik. und diätet. Therapie igio.

I7. $\mathrm{Pu}$ s e y, A., The use of carbon dioxide snow in the treatment of naevi and other lesions of the skin. Journ. of the Amer. Med. Assoc. I907, Oct. 19.

I8. Derselbe, Kohlensäureschnee zur Behandlung von Hautkrankheiten. Berl. klin. Wochenschr. I908, Nr. 24.

I9. Derselbe, The therapeutic use of solidified carbon dioxide. Ann. meet. of the Pittsburg Acad. of Medicine I908, Dec. I9.

2o. Derselbe, The therapeutic use of refrigeration, particularly with solid carbon dioxide. Journ. of cutan. diseases igio, July.

2I. Salomon, Anatomische und klinische Beiträge zur Behandlung der Angiome und Naevi mittels Kohlensäureschnee. Deutsche Zeitschr. für Chir. I9тा, Bd. I09, S. 518.

22. S a u e r b u c h, F., Die Behandlung der Angiome mit gefrorener Kohlensäure. Zentralbl. für Chir. I909, Nr. I.

23. S to ut, Naevus pigmentosus, treated with carbon dioxide. Journ. cut. dis. I909, Bd. 27 , S. 266.

24. S t r a u B, A., Die Behandlung der Naevi mit Kohlensäureschnee. Deutsche med. Wochenschrift 1908 , Nr. 53 .

25. Derselbe, Die Technik der Kohlensäureschneebehandlung bei Hautkrankheiten. Münchn. med. Wochenschr. IgII, Bd. I.

26. D e r s elb e , „Tubus zur Behandlung mit Kohlensäureschnee“. Erwiderung an Kuznitsky. Münch. Med. Wochenschr. Igr I, Nr. I4.

27. Sutton, R., The use of carbon dioxide snow in dermatology. Dublin, Journal of Med. Sc. Igog, July ig.

28. $Z$ e is l e r, J., Observations on the use of liquid carbon dioxide. Journ. of cut. diseases I909, Bd. 27, S. 32 .

29. Z weig, L., Die Behandlung von umschriebenen Hauterkrankungen mit Kohlensäureschnee. Münch. med. Wochenschr. I909, Nr. 22, S. I643. 